\title{
Late-time Optical Emission From Core-collapse Supernovae
}

\section{Citation}

Milisavljevic, Dan, Robert A. Fesen, Roger A. Chevalier, Robert P. Kirshner, Peter Challis, and Massimo Turatto. 2012. "LATE-TIME OPTICAL EMISSION FROM CORE-COLLAPSE SUPERNOVAE." The Astrophysical Journal 751 (1): 25. https:// doi.org/10.1088/0004-637x/751/1/25.

\section{Permanent link}

http://nrs.harvard.edu/urn-3:HUL.InstRepos:41399790

\section{Terms of Use}

This article was downloaded from Harvard University's DASH repository, and is made available under the terms and conditions applicable to Other Posted Material, as set forth at http:// nrs.harvard.edu/urn-3:HUL.InstRepos:dash.current.terms-of-use\#LAA

\section{Share Your Story}

The Harvard community has made this article openly available. Please share how this access benefits you. Submit a story.

Accessibility 


\title{
LATE-TIME OPTICAL EMISSION FROM CORE-COLLAPSE SUPERNOVAE
}

\author{
Dan Milisavljevic ${ }^{1,5}$, Robert A. Fesen ${ }^{1}$, Roger A. Chevalier ${ }^{2}$, Robert P. Kirshner ${ }^{3}$, \\ Peter Challis ${ }^{3}$, and Massimo Turatto ${ }^{4}$ \\ ${ }^{1} 6127$ Wilder Lab, Department of Physics \& Astronomy, Dartmouth College, Hanover, NH 03755, USA; dmilisav@cfa.harvard.edu \\ ${ }^{2}$ Department of Astronomy University of Virginia, P.O. Box 400325, Charlottesville, VA 22904-4325, USA \\ ${ }^{3}$ Harvard-Smithsonian Center for Astrophysics, 60 Garden Street, Cambridge, MA 02138, USA \\ ${ }^{4}$ Osservatorio Astronomico di Padova, vicolo dell'Osservatorio 5, I-35122 Padova, Italy \\ Received 2011 September 8; accepted 2012 February 29; published 2012 May 1
}

\begin{abstract}
Ground-based optical spectra and Hubble Space Telescope images of 10 core-collapse supernovae (CCSNe) obtained several years to decades after outburst are analyzed with the aim of understanding the general properties of their late-time emissions. New observations of SN 1957D, 1970G, 1980K, and 1993J are included as part of the study. Blueshifted line emissions in oxygen and/or hydrogen with conspicuous line substructure are a common and long-lasting phenomenon in the late-time spectra. Followed through multiple epochs, changes in the relative strengths and velocity widths of the emission lines are consistent with expectations for emissions produced by interaction between SN ejecta and the progenitor star's circumstellar material. The most distinct trend is an increase in the strength of $[\mathrm{O} \mathrm{III}] /\left([\mathrm{O} \mathrm{I}]+\left[\mathrm{O}_{\mathrm{II}}\right]\right)$ with age, and a decline in $\mathrm{H} \alpha /([\mathrm{O} \mathrm{I}]+[\mathrm{O} \mathrm{II}])$ which is broadly consistent with the view that the reverse shock has passed through the $\mathrm{H}$ envelope of the ejecta in many of these objects. We also present a spatially integrated spectrum of the young Galactic supernova remnant Cassiopeia A (Cas A). Similarities observed between the emission line profiles of the $\approx 330 \mathrm{yr}$ old Cas A remnant and decades old CCSNe suggest that observed emission line asymmetry in evolved CCSN spectra may be associated with dust in the ejecta, and that minor peak substructure typically interpreted as "clumps" or "blobs" of ejecta may instead be linked with large-scale rings of $\mathrm{SN}$ debris.
\end{abstract}

Key words: ISM: individual objects (Cassiopeia A) - ISM: supernova remnants - supernovae: individual (SN 1957D, SN 1970G, SN 1980K, SN 1993J) - supernovae: general

Online-only material: color figures

\section{INTRODUCTION}

Optical spectra of core-collapse supernovae (CCSNe) beyond a couple years after maximum light are difficult to obtain due to their increasing faintness with time and thus are relatively rare. Given that the majority of CCSNe occur at distances $>10 \mathrm{Mpc}$ and fade at least 8 mag below peak brightness within their first two years (Kirshner 1990), observations have been largely limited to the first 700 days or so after maximum light when they are at apparent magnitudes $\lesssim 20$.

However, in a handful of cases it has been possible to monitor CCSNe several years or even decades post-outburst. This may be because of a fortuitously nearby distance, such as SN 1987A in the Large Magellanic Cloud $(D \sim 50 \mathrm{kpc}$; Kunkel et al. 1987; Feast 1999; van Leeuwen et al. 2007), or exceptional circumstances wherein some late-time energy source maintains optical luminosity at observable levels. This latter scenario was first recognized in the late 1980s with the optical re-detections of SN 1980K (Fesen \& Becker 1988) and SN 1957D (Long et al. 1989; Turatto et al. 1989).

Of the various late-time mechanisms theorized to power CCSN emission at epochs $>2 \mathrm{yr}$, the most common and bestunderstood process is the forward shock front and SN ejecta interaction with surrounding circumstellar material (CSM) shed from the progenitor star (see Chevalier \& Fransson 2003, 2006 and references therein). SN-CSM interaction emits across a wide spectral band spanning radio to X-ray. Optical emissions largely originate from a reverse shock that propagates upstream into outward expanding ejecta that gets heated and ionized (Chevalier \& Fransson 1994). The most dominant emission

\footnotetext{
5 Now at Harvard-Smithsonian Center for Astrophysics, 60 Garden Street, Cambridge, MA 02138, USA.
}

lines are [OI] $\lambda \lambda 6300,6364,[\mathrm{O}$ II $] \lambda \lambda 7319,7330,[\mathrm{O}$ III] $\lambda \lambda 4959,5007$, and $\mathrm{H} \alpha$ with broad linewidths $\gtrsim 2000 \mathrm{~km} \mathrm{~s}^{-1}$. Other proposed late-time mechanisms include pulsar/magnetar interaction with expanding SN gas (Chevalier \& Fransson 1992; Kasen \& Bildsten 2010; Woosley 2010) or accretion onto a black hole remnant (Patnaude et al. 2011).

Studies of late-time optical emissions can yield kinematic and chemical information about the SN ejecta and probe the mass-loss history and evolutionary status of the progenitor stars (Leibundgut et al. 1991; Fesen et al. 1999). The evolution of line widths and differences between the relative line strengths can distinguish between late-time mechanisms and be important diagnostics of the ejecta structure.

Here, we present optical images and spectra of $10 \mathrm{CCSNe}$ observed during the relatively unexplored late-time transition phase between SN outburst and SN remnant formation. In Sections 2 and 3, we present and briefly discuss high-resolution images and new low-resolution optical spectra of SN 1957D, SN 1970G, SN 1980K, and SN 1993J along with archival latetime spectra of six other CCSNe. Common properties observed in these data are reviewed in Section 4 and then interpreted in the context of an SN-CSM interaction model in Section 5. Further investigation of these spectra follow from comparing them to a spatially integrated spectrum of the young Galactic supernova remnant (SNR) Cassiopeia A in Section 6. A summary of our findings is given in Section 7.

\section{OBSERVATIONS}

\subsection{Images}

High-resolution images obtained with the Hubble Space Telescope (HST) covering the sites of SN 1957D, 1970G, 
Table 1

Summary of $H S T$ Images

\begin{tabular}{|c|c|c|c|c|c|c|c|}
\hline Inst. & Filter & $\begin{array}{l}\lambda_{\text {cen }} \\
(\AA)\end{array}$ & $\begin{array}{c}\lambda_{\mathrm{FWHM}} \\
(\AA)\end{array}$ & $\begin{array}{c}\text { Descriptive } \\
\text { Notes }\end{array}$ & $\begin{array}{l}\text { Date } \\
\text { (UT) }\end{array}$ & $\begin{array}{l}\text { Exp Time } \\
\text { (s) }\end{array}$ & Program No./PI \\
\hline \multicolumn{8}{|c|}{ SN 1957D } \\
\hline \multirow[t]{6}{*}{ WFC3 } & F336W & 3375 & 550 & Johnson $U$ & 2010 Mar 17 & 2560 & 11360/R. O'Connell \\
\hline & F438W & 4320 & 695 & Johnson $B$ & 2010 Mar 17 & 1800 & \\
\hline & F502N & 5013 & 47 & [O III] & 2010 Mar 19 & 2484 & \\
\hline & F547M & 5475 & 710 & Continuum & 2010 Mar 20 & 1203 & \\
\hline & F657N & 6573 & 94 & $\mathrm{H} \alpha+[\mathrm{N}$ II $]$ & 2010 Mar 19 & 1484 & \\
\hline & F673N & 6731 & 77 & {$[\mathrm{~S} \mathrm{II}]$} & 2010 Mar 17 & 1770 & \\
\hline \multicolumn{8}{|c|}{ SN 1970G } \\
\hline \multirow[t]{2}{*}{ WFPC2 } & F606W & 5997 & 1502 & Wide- $V$ & 1998 Apr 21 & 600 & 6713/W. Sparks \\
\hline & F656N & 6564 & 22 & $\mathrm{H} \alpha$ & 1998 Apr 21 & 1600 & \\
\hline \multicolumn{8}{|c|}{ SN 1980K } \\
\hline \multirow[t]{2}{*}{ WFPC2 } & F606W & 5997 & 1502 & Wide- $V$ & 2008 Jan 19 & 1600 & 11229/M. Meixner \\
\hline & F814W & 7940 & 1531 & Johnson $I$ & 2008 Jan 19 & 1600 & \\
\hline
\end{tabular}

and $1980 \mathrm{~K}$ were retrieved from the Multimission Archive at Space Telescope (MAST) and Hubble Legacy Archive (HLA) maintained by the Space Telescope Science Institute (STScI) to examine the supernovae and their local environments. Table 1 lists the instruments, filter passbands with notes about their emission line sensitivies, dates, exposure times, and program details of the observations. All images retrieved from MAST were manually co-added and cleaned of cosmic rays using the crrej and multidrizzle tasks in IRAF/PyRAF, ${ }^{6}$ whereas those retrieved from the HLA were automatically drizzled by the archive through pipelined software.

\subsection{Spectra}

Late-time, low-dispersion optical spectra of four CCSNe were obtained using a variety of telescopes and instrumental setups. Below we describe the details of these observations.

Spectra of SN 1957D were obtained on 2001 July 27 with the FOcal Reducer/low dispersion Spectrograph 1 (FORS1) at the ESO Very Large Telescope (VLT) at Paranal, Chile. The CCD detector has a scale of 0.2 pixel $^{-1}$, and the GRIS300V grism with a GG435 filter was used with a $1^{\prime \prime}$ slit to produce spectra ranging from 4400-8700 $\AA$ with FWHM resolution of approximately $10 \AA$. Total exposure time was $1800 \mathrm{~s}$ obtained at an airmass of 1.7. An atmospheric dispersion corrector minimized potential effects of atmospheric refraction.

Observations of SN 1970G were obtained on 2010 March 12 with the $2.4 \mathrm{~m}$ Hiltner telescope at MDM Observatory on Kitt Peak, Arizona. A Boller \& Chivens CCD spectrograph was used with a north-south $1^{\prime \prime} .5 \times 5^{\prime}$ slit, a 150 lines $\mathrm{mm}^{-1}$ $4700 \AA$ blaze grating, and an LG400 filter to block contaminating second-order light. Exposures totaling $2 \times 3600 \mathrm{~s}$ obtained at culmination at airmasses $<1.1$ were combined. Resulting spectra spanned 4300-7900 $\AA$ with resolution of $11 \AA$. Conditions were mostly photometric with the seeing around 1 1".2.

The $2.4 \mathrm{~m}$ Hiltner telescope at MDM was also used to obtain spectra of SN 1980K on 2010 October 9. The Mark III Spectrograph with a SITe $1024 \times 1024$ CCD detector was used.

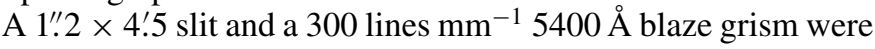

\footnotetext{
6 IRAF is distributed by the National Optical Astronomy Observatory, which is operated by the Association of Universities for Research in Astronomy, Inc. under cooperative agreement with the National Science Foundation. PyRAF is a product of the Space Telescope Science Institute, which is operated by AURA for NASA.
}

employed. A total of $2 \times 3000 \mathrm{~s}$ exposures were obtained at culmination around an airmass of 1.1 with a spectral resolution of $7 \AA$. Conditions were photometric with subarcsecond seeing.

Spectra of SN 1993J were obtained on 2009 December 9 with the $6.5 \mathrm{~m}$ MMT at Mt. Hopkins in Arizona using the HECTOSPEC optical fiber fed spectrograph. These observations were part of a survey of SNRs of M81. Spectra from the 1'.5 diameter fibers cover the wavelength range of 3700-9200 with FWHM resolution of $5 \AA$. Observations were obtained at an airmass of 1.3 with an atmospheric dispersion corrector in place and the total exposure time was $3600 \mathrm{~s}$.

These spectra were reduced and calibrated employing standard techniques in IRAF and our own IDL routines (see Matheson et al. 2008). Cosmic rays and obvious cosmetic defects have been removed from all spectra. Wavelengths were checked against night sky emission lines. Flux calibrations were from observations of Stone (1977) and Massey \& Gronwall (1990) standard stars.

\section{LATE-TIME CCSN SPECTRA AND IMAGES}

\subsection{SN 1957D}

SN 1957D in M83 ( $D \sim 4.6 \mathrm{Mpc}$; Saha et al. 2006) was discovered by H. Gates on 1957 December 28 when the SN was well past maximum light in a spiral arm about $3^{\prime} \mathrm{NNE}$ of the galaxy nucleus (Gates \& Carpenter 1958). The SN was followed photometrically on 1958 January 16 and 30, and February 14 when its photographic magnitude was 16.7 . No spectrum was obtained during this early period.

Approximately $25 \mathrm{yr}$ after outburst, the $\mathrm{SN}$ was recovered as a radio source (Cowan \& Branch 1982; Pennington \& Dufour 1983). Subsequent observations by Long et al. (1989) and Turatto et al. (1989) showed that its optical spectra were dominated by broad [O III] $\lambda \lambda 4959,5007$ lines. Additional follow-up observations by Long et al. (1992) showed that the optical flux had decreased by at least a factor of five between 1987 and 1991, and Cappellaro et al. (1995) confirmed the rapid decline. Pennington et al. (1982) suggested that the SN was produced by a massive Population I progenitor based on integrated colors in the surrounding region, corroborating suspicion that this was a Type II or Ib/c event (Long et al. 1989). 


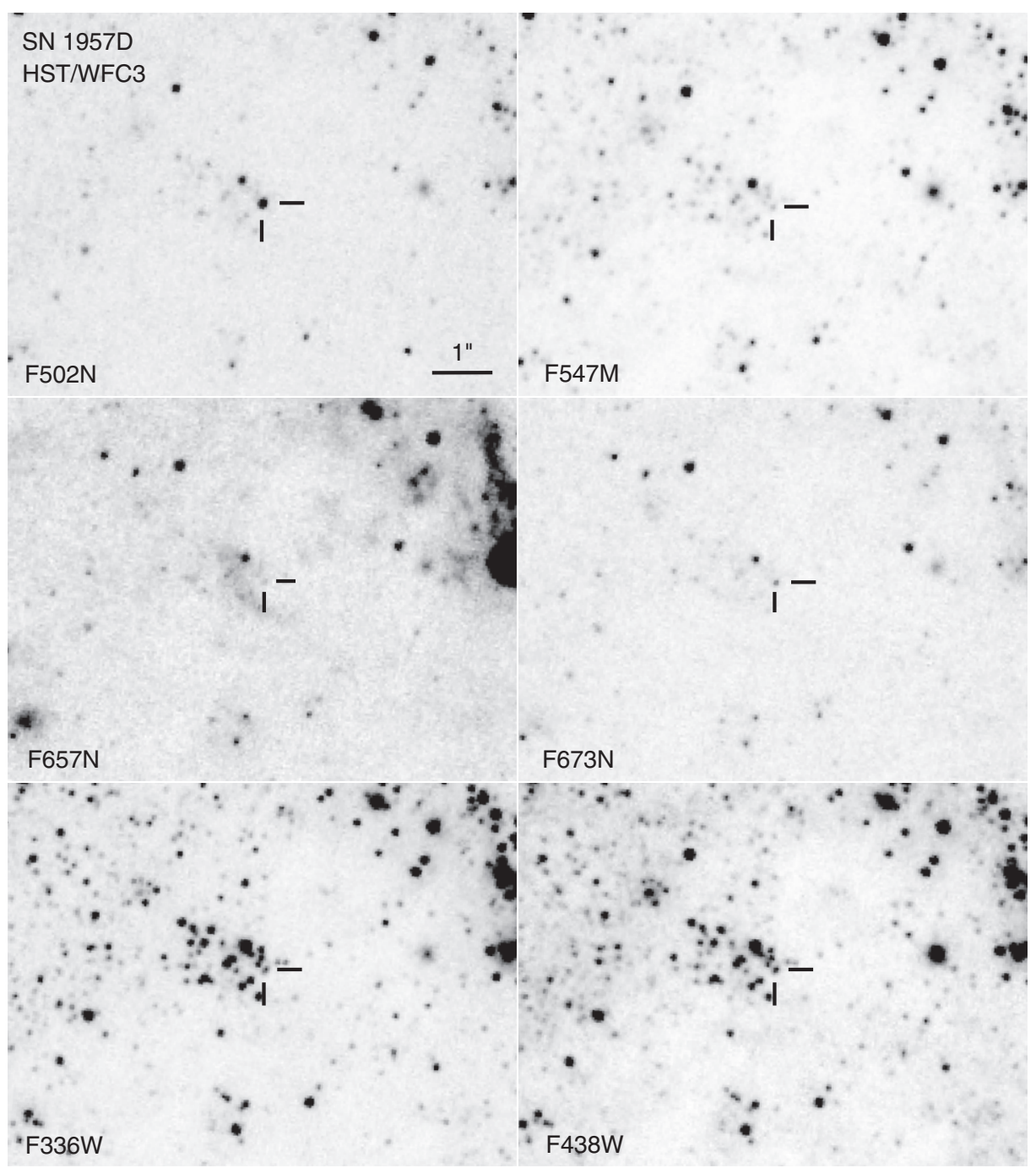

Figure 1. HST WFC3/UVIS images of the region around SN 1957D obtained in 2010 March. The location of the SN is marked. For these images and all those that follow, north is up and east is to the left.

In Figure 1, we show high-resolution $H S T$ images of the region around SN 1957D which provide the first clear look at the supernova's neighboring stellar environment. The O-rich SN is bright and unresolved in the F502N image. We estimate an [O III] $\lambda 5007$ flux of $(7.8 \pm 0.3) \times 10^{-16} \mathrm{erg} \mathrm{s}^{-1} \mathrm{~cm}^{-2}$, which implies a decline from 1991 April when it was $1.8 \times 10^{-15} \mathrm{erg} \mathrm{s}^{-1} \mathrm{~cm}^{-2}$ (Long et al. 1992). The SN is marginally detected in the $\mathrm{H} \alpha$-sensitive $\mathrm{F} 657 \mathrm{~N}$ image where we see its location just outside a nearby $\mathrm{H}$ II region. The $\mathrm{SN}$ is also visible in the [S II]-sensitive F673N image with emission of the order of $1 \times 10^{-16} \mathrm{erg} \mathrm{s}^{-1} \mathrm{~cm}^{-2}$. Emission is stronger in the F673N image than in the F657N image, suggesting [S II] $\lambda \lambda 6716,6731$ line emission associated with shocked $\mathrm{SN}$ ejecta.

The F336W and F438W images indicate that the remnant is projected along the outskirts of a cluster of blue stars with dimensions of approximately $1^{\prime \prime} \times 1$ 1.5 or $25 \times 35$ $(\mathrm{d} / 4.6 \mathrm{Mpc}) \mathrm{pc}$. Emission in these filter images, bright in the $U$ and $B$ passbands, is seen at the location of the SN, likely associated with nearby massive stars. The stellar environment of SN 1957D resembles the luminous SNR in NGC 4449 as observed with $H S T$ (SNR 4449-1; Milisavljevic \& Fesen 2008). This is another O-rich, young remnant with an age of $\sim 70 \mathrm{yr}$ estimated from the ratio of angular size to expansion velocity (Bietenholz et al. 2010).

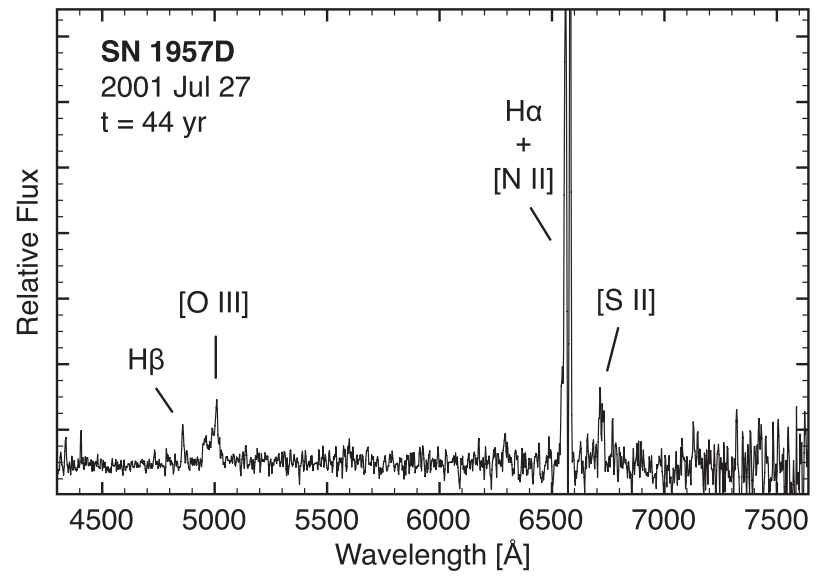

Figure 2. Optical spectrum of SN 1957D obtained with the VLT on 2001 July 27. Broad [O III] $\lambda \lambda 4959,5007$ emission originates from the $\mathrm{SN}$, while the narrow $\mathrm{H} \alpha,[\mathrm{N} \mathrm{II}] \lambda \lambda 6548,6583, \mathrm{H} \beta$, and [S $\mathrm{II}] \lambda \lambda 6716,6731$ lines are associated with a nearby $\mathrm{H}$ II region (see Figure 1).

In Figure 2, we present a 2001 optical spectrum of SN 1957D, some $44 \mathrm{yr}$ after outburst. Wavelengths have been corrected for the host galaxy's redshift of $513 \mathrm{~km} \mathrm{~s}^{-1}$ and a blue continuum associated with background starlight has been subtracted using a 


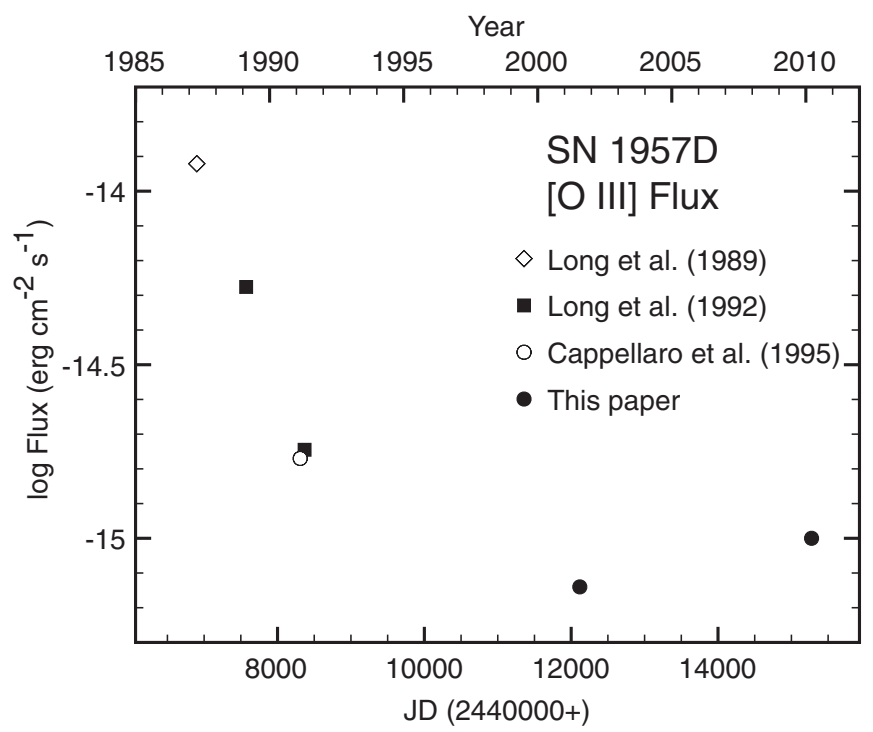

Figure 3. History of reported [O III] $\lambda \lambda 4959,5007$ fluxes for SN 1957D. All measurements are from the spectral lines except for the 2010.3 HST observation which is from a narrow band [O III] $\lambda 5007$ image and has been corrected to compensate for the unsampled $\lambda 4959$ line.

third-order Chebyshev function. Narrow $\mathrm{H} \alpha, \mathrm{H} \beta$, [N II] $\lambda \lambda 6548$, 6583 , and [S II] $\lambda \lambda 6716,6731$ line emissions are associated with the nearby $\mathrm{H}$ II region, and the sharp cut in the $\mathrm{H} \alpha$ profile is due to poor subtraction of this emission. The dominant emission lines observed from the SN ejecta are broad [O III] $\lambda \lambda 4959$, 5007.

The half-width at zero intensity (HWZI) of the [O III] emission is approximately $1000 \mathrm{~km} \mathrm{~s}^{-1}$, confirmed by measuring both from $5007 \AA$ to the red and $4959 \AA$ toward the blue. The measured [O III] $\lambda \lambda 4959,5007$ flux is $(7.1 \pm 0.5) \times$ $10^{-16} \mathrm{erg} \mathrm{s}^{-1} \mathrm{~cm}^{-2}$. Accounting for possible slit losses, this measurement is consistent with the decline between the Long et al. (1992) and the 2010 HST/WFC3 F502N image measurements. In Figure 3, we plot the handful of published [O III] fluxes. A clear decrease in flux is seen between previously reported values from Long et al. (1992) and Cappellaro et al. (1995) and the 2001 and 2010 measurements presented here. The last two measurements give the impression that the decline in flux has slowed or even leveled out.

In Figure 4, we show a comparison of the 2001 July [O III] line profile of SN 1957D to a spectrum obtained in 1989 April (Turatto et al. 1989). Some narrowing of the emission line of order $-1000 \mathrm{~km} \mathrm{~s}^{-1}$ may have occurred between the $10 \mathrm{yr}$ that separates the observations $\left(\sim 100 \mathrm{~km} \mathrm{~s}^{-1} \mathrm{yr}^{-1}\right)$ although the signal-to-noise ratio $(\mathrm{S} / \mathrm{N})$ prevents a firm conclusion. Our 1989 measurement is in agreement with Long et al. (1992) who reported [O III] emission line velocities in excess of $\pm 2000 \mathrm{~km} \mathrm{~s}^{-1}$ in their optical spectra taken at a similar epoch.

\subsection{SN $1970 G$}

SN 1970G in M101 ( $D \sim 6.7$ Mpc; Freedman et al. 2001) was discovered by M. Lovas on 1970 July 30 (Detre \& Lovas 1970) near maximum light $(B=11.5$; Winzer 1974; Barbon et al. 1979) along the NW boundary of the galaxy's large H II complex NGC 5455. Optical spectra showed Type II SN features with broad $\mathrm{H} \alpha$ emission (Kirshner et al. 1973; Kirshner \& Kwan 1974). It has been generally classified as a Type IIL based on its linearly declining light curve (Barbon et al. 1979; Young $\&$ Branch 1989), but a brief plateau phase between day 30

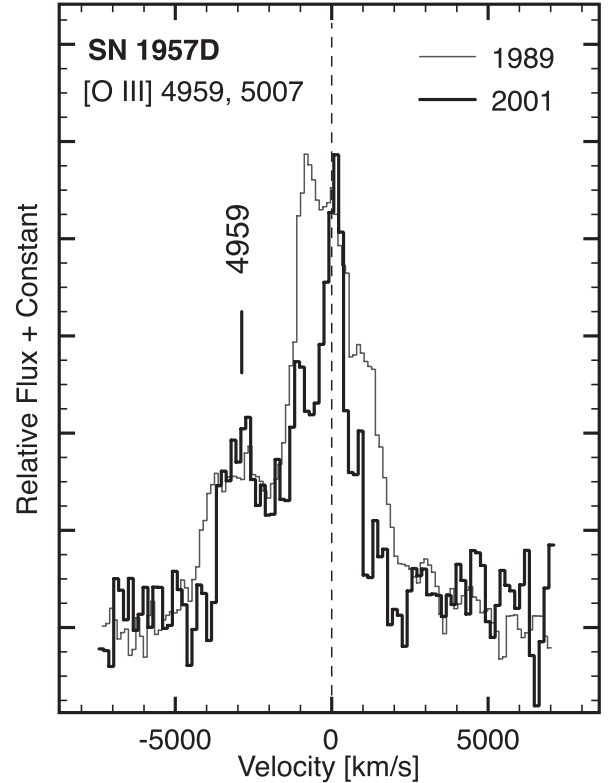

Figure 4. Emission line profiles of SN 1957D's [O III] $\lambda \lambda 4959,5007$ emission in 1989 and 2001. Velocities are with respect to $5007 \AA$.

and 50 and optical spectra exhibiting prominent P-Cygni line absorption features may make it a transitional object between IIP and IIL (Barbon et al. 1979; Turatto et al. 1990).

SN 1970G was the first supernova to be detected in the radio and remains the longest monitored radio supernova (Stockdale et al. 2001). Radio flux densities were relatively constant for three years before beginning to fade in 1974 (Allen et al. 1976; Brown \& Marscher 1978; Weiler et al. 1986). Radio emission was re-detected in 1991 by Cowan et al. (1991), prompting optical observations by Fesen (1993) who obtained spectra revealing broad $\mathrm{H} \alpha$ and $\left[\mathrm{O}_{\mathrm{I}}\right] \lambda \lambda 6300,6364$ emissions.

Two optical HST images of the region around SN $1970 \mathrm{G}$ obtained in 1998 April are shown in Figure 5. To the south lies the large H II region NGC 5455. The F606W image shows three sources around the radio coordinates $\alpha(2000)=14^{\mathrm{h}} 03^{\mathrm{m}} 00.88$ $\delta(2000)=+54^{\circ} 14^{\prime} 33^{\prime \prime}$. 1 (Stockdale et al. 2001) centered in the figure. Although a finding chart published in Fesen (1993) suggests the brighter, eastern source as the $\mathrm{SN}$, alignment of these images with a CFHT MegaPipe image of the region (Group G002.308.620+60.034) having astrometric accuracy $<0$.' 1 favors the western source and is the object marked in Figure 5. The observed F606W/F656N flux ratio of this object is in agreement with the spectrum of the $\mathrm{SN}$ which shows broad $\mathrm{H} \alpha$ emission.

In the top panel of Figure 6, we show a 2010 optical spectrum of SN 1970G some $40 \mathrm{yr}$ after outburst. Wavelengths have been corrected for the host galaxy's redshift of $251 \mathrm{~km} \mathrm{~s}^{-1}$ and an underlying blue continuum believed to be associated with background starlight has been subtracted with a third-order Chebysev function. The spectrum is dominated by narrow H II region lines of [O III], $\mathrm{H} \alpha, \mathrm{H} \beta,[\mathrm{N} \mathrm{II}] \lambda \lambda 6548,6583$, and [S II] $\lambda \lambda 6716,6731$. Noise around $7300 \AA$ is due to poor night sky $\mathrm{OH}$ subtraction.

An enlarged portion of the spectrum with the $\mathrm{H}$ II region lines manually removed is shown in the lower panel of Figure 6 in order to better see the faint underlying emission originating from the supernova. $\mathrm{H} \alpha$ is the strongest emission line and exhibits a mildly asymmetric profile shifted blueward. There is no clear change from the profile described in Fesen (1993). 


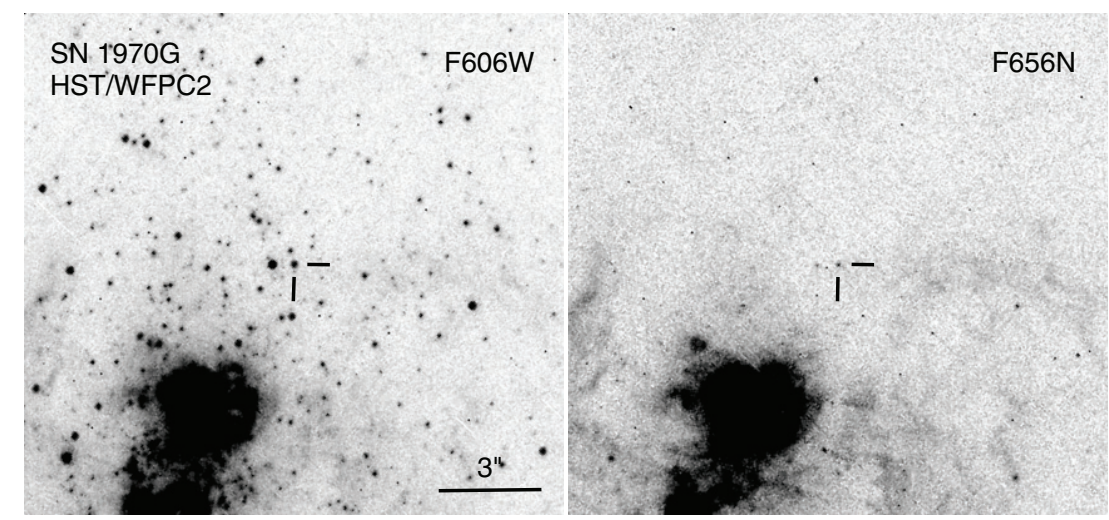

Figure 5. HST/WFPC2 images of the region around SN 1970G obtained on 1998 April 21. Left: F606W (Wide V) image in high contrast marking SN 1970G as one of the brightest objects in the field. Right: F656N image sensitive to $\mathrm{H} \alpha$.
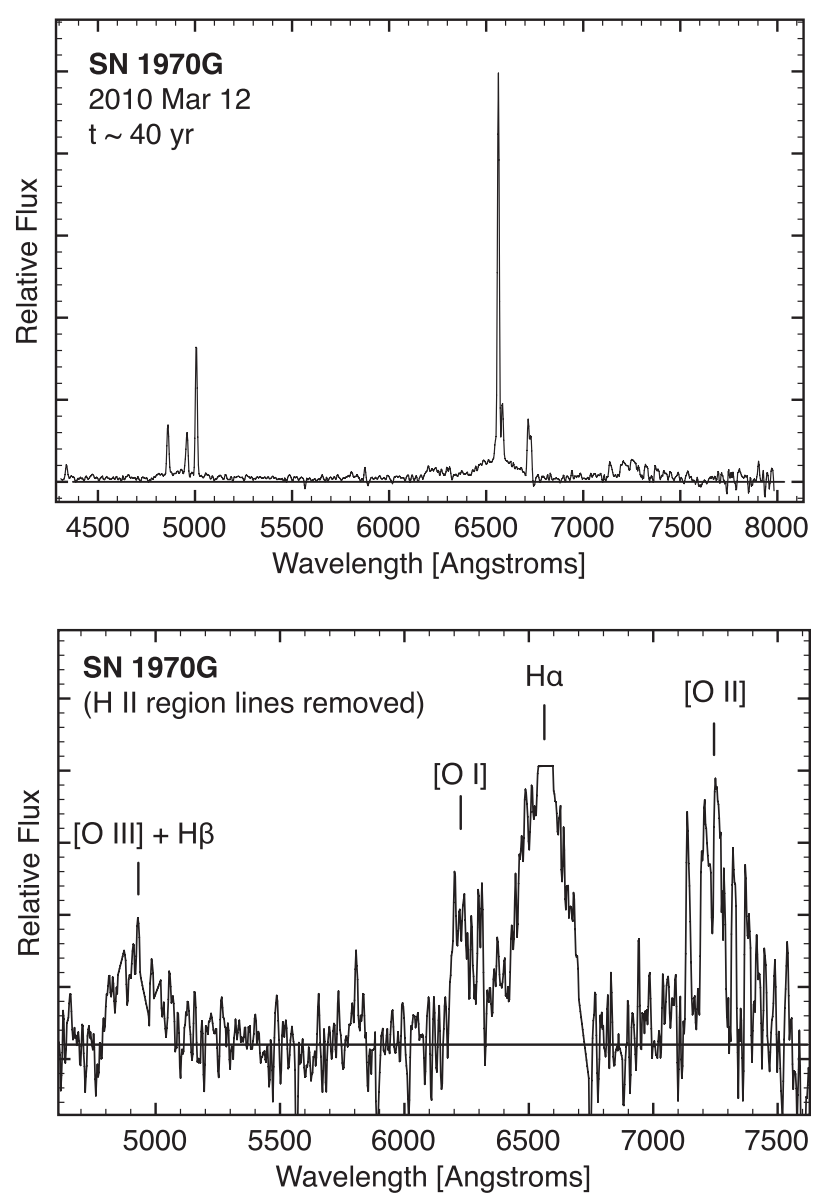

Figure 6. Top: MDM optical spectrum of SN 1970G 40 yr post-outburst. The narrow emission lines dominating the spectrum are from a coincident $\mathrm{H}$ II region. Bottom: enlarged spectrum with $\mathrm{H}$ II region lines removed and line identifications marked. A blue continuum has been subtracted.

The estimated $\mathrm{H} \alpha$ flux (minus background and narrow $\mathrm{H}$ II region emission) is $(1.7 \pm 0.4) \times 10^{-15} \mathrm{erg} \mathrm{s}^{-1} \mathrm{~cm}^{-2}$, relatively unchanged from $1.8 \times 10^{-15} \mathrm{erg} \mathrm{s}^{-1} \mathrm{~cm}^{-2}$ measured $18 \mathrm{yr}$ earlier (Fesen 1993). The width of $\mathrm{H} \alpha$ is estimated to span $6410 \AA$ to $6690 \AA\left(-7000\right.$ to $\left.+5800 \mathrm{~km} \mathrm{~s}^{-1}\right)$ with uncertainty perhaps greater than $\sim 500 \mathrm{~km} \mathrm{~s}^{-1}$ due to confusion with the broad [O I] $\lambda \lambda 6300,6364$ and narrow [S II] $\lambda \lambda$ 6716, 6731 lines. The velocity width measured from data obtained in 1992 was reported to extend from $-5200 \mathrm{~km} \mathrm{~s}^{-1}$ to $+5600 \mathrm{~km} \mathrm{~s}^{-1}$, but this change is likely the result of improvement in $\mathrm{S} / \mathrm{N}$.
Broad emission spanning 4780-5090 Å showing a strongly blueshifted, gradually descending profile is detected. We associate the emission primarily with [O III], but given the large implied blueshifted velocity $\left(<-10^{4} \mathrm{~km} \mathrm{~s}^{-1}\right)$ with respect to the $4959 \AA$ line, contribution from $\mathrm{H} \beta$ is likely.

[OI] emission shows the same gradually descending profile as [O III]. We measure a velocity extension from $6175 \AA$ $\left(-6000 \mathrm{~km} \mathrm{~s}^{-1}\right.$ with respect to $\left.6300 \AA\right)$ to $6410 \AA\left(+2200 \mathrm{~km} \mathrm{~s}^{-1}\right.$ with respect to $6364 \AA$ ) where it merges with the broad $\mathrm{H} \alpha$. Broad emission centered around $7240 \AA$ could be associated with the $[\mathrm{Ca}$ II] $\lambda \lambda 7291,7324$ and/or [O II] $\lambda \lambda 7319,7330$ lines (Fesen 1993). Because [O II] is known to be a dominant line in intermediate-aged supernovae (see discussion in Fesen et al. 1999) and the velocity line profile best matches the [O I] distribution when centered with respect to $7325 \AA$, we identify [O II] as the major contributor.

Faint emission centered around $5810 \AA$ having a full width at zero intensity less than $100 \AA$ is also weakly detected. It was not detected in the Fesen (1993) spectrum and may be associated with blueshifted $\mathrm{He}$ I and/or Na I emission.

\subsection{SN $1980 K$}

SN 1980K in NGC 6946 ( $D \sim 5.9$ Mpc; Karachentsev et al. 2000) was discovered by P. Wild on 1980 October 28 and reached a peak brightness of $V=11.4$ in 1980 November (Buta 1982). Photographic observations showing an almost linear decline in brightness and spectra revealing broad $\mathrm{H} \alpha$ with minor P Cygni absorption classify it as a Type IIL (Barbon et al. 1982). Radio and X-ray emissions were detected about a month after maximum (Canizares et al. 1982; Weiler et al. 1986). Radio emissions were followed quite extensively through 1996 (Weiler et al. 1992; Montes et al. 1998) and more limited X-ray observations have been obtained through 2004 (Schlegel 1994; Soria \& Perna 2008).

The SN declined steadily in the optical through 1982 (Uomoto \& Kirshner 1986) but was detected in 1987 nearly seven years after maximum through narrow passband imaging (Fesen \& Becker 1988). Follow-up low-dispersion optical spectra showed broad $\mathrm{H} \alpha$ and [O I] emission along with weaker line emission from [O III], [Fe II] $\lambda 7155$, and emission around $7300 \AA$ identified as [Ca II] $\lambda \lambda 7291,7324$ and/or [O II] $\lambda \lambda 7319,7330$ (Fesen \& Becker 1990; Uomoto 1991; Leibundgut et al. 1991). Further monitoring through 1997 indicated no major changes in the spectrum aside from steadily declining $\mathrm{H} \alpha$ emission (Leibundgut et al. 1993; Fesen \& Matonick 1994; Fesen et al. 1999). 


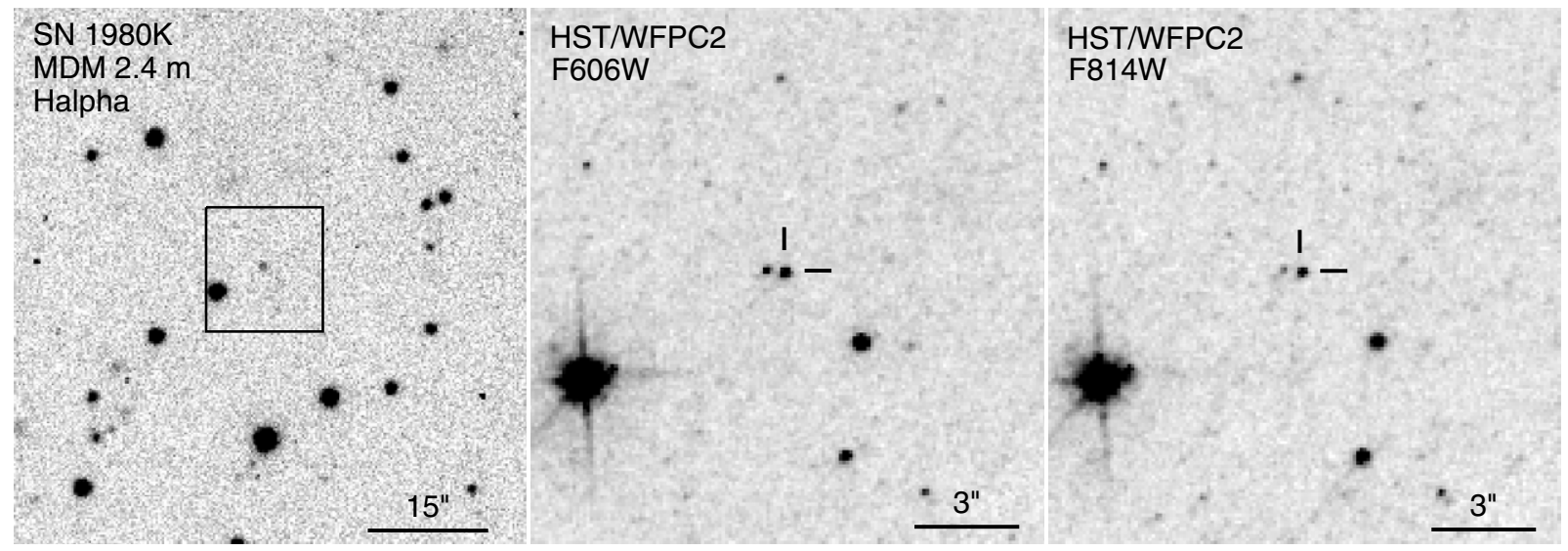

Figure 7. Optical images of SN 1980K. Left: $\mathrm{H} \alpha$ image obtained in 1997 May with the $2.4 \mathrm{~m}$ Hiltner telescope showing the environment around the SN. The boxed region demarcates the enlarged portion shown in other panels. Middle and right: HST/WFPC2 images obtained in 2008 January with SN 1980K marked.

High-resolution HST images obtained in 2008 January (Figure 7) show the environment of SN 1980K in detail never seen before. Two unresolved sources around the published coordinates of the supernova $\alpha(2000)=20^{\mathrm{h}} 35^{\mathrm{m}} 30^{\mathrm{s}} .07 \delta(2000)=$ $+60^{\circ} 06^{\prime} 23^{\prime \prime} 8$ (van Dyk et al. 1996) are seen. We compared the images to an unpublished ground-based $\mathrm{H} \alpha$ image $\left(\lambda_{\mathrm{c}}=\right.$ $6564 \AA ; \Delta \lambda=30 \AA$ ) of the region obtained by R. Fesen on 1997 May 8 using the Hiltner $2.4 \mathrm{~m}$ telescope at MDM Observatory (also shown in Figure 7) to confirm that the supernova was the brighter, more westerly source. The other source, approximately 0'.55 away or $15(\mathrm{~d} / 5.9 \mathrm{Mpc}) \mathrm{pc}$, has a rather steep color difference between the F606W and F814W images suggestive of a luminous blue star or very tight stellar association.

The top panel of Figure 8 shows our recent 2010 spectrum of SN 1980K with identified emission lines marked. The small recession velocity of $40 \mathrm{~km} \mathrm{~s}^{-1}$ of NGC 6946 has been corrected. A weak blue continuum that trails off around $5500 \AA$ may be due to light contamination from the projected companion seen slightly east of the SN in the HST/WFC2 images (Figure 7).

The $\mathrm{H} \alpha$ and [OI] lines exhibit broad, asymmetric emission profiles strongly blueshifted with an emission peak around $-2900 \mathrm{~km} \mathrm{~s}^{-1}$. Emission around $7300 \AA$ associated with [O II] (Fesen et al. 1999) is weakly detected. Asymmetric emission spanning $4935-5010 \AA$ is identified as strongly blueshifted [O III]. A narrow unresolved emission peak around $6558 \AA$ A , seen in earlier spectra, is potentially due to a small $\mathrm{H}$ II region near the SN site or ionized wind material associated with the progenitor (Fesen \& Becker 1990; Fesen et al. 1995). Another peak around $5003 \AA$ is also observed; if associated with [O III] $\lambda 5007$, this is at the same $\approx-200 \mathrm{~km} \mathrm{~s}^{-1}$ blueshifted velocity of the narrow $\mathrm{H} \alpha$ line. Emission around $7090 \AA$ As likely associated with [Fe II] $\lambda 7155$ blueshifted $-2700 \mathrm{~km} \mathrm{~s}^{-1}$, but some contribution from [Ar III] $\lambda 7136$ is possible.

The bottom of Figure 8 compares our 2010 spectrum of SN 1980K with Keck spectra obtained in 1995 (Fesen et al. 1999). [O I] and $\mathrm{H} \alpha$ show minor changes to their line profiles and overall reduction in emission strength, while the flux ratio of $[\mathrm{O}$ III $] / \mathrm{H} \alpha$ has increased. The overall [O II] flux has decreased, but the true extent of diminishment is difficult to gauge firmly because of low $\mathrm{S} / \mathrm{N}$ and poor night sky subtraction.

The $\mathrm{H} \alpha$ line profile has a velocity width of -5300 to $+6000 \mathrm{~km} \mathrm{~s}^{-1}$. The blueshifted emission width is narrower than the 1995 measurement of $-5700 \mathrm{~km} \mathrm{~s}^{-1}$, and the redshifted width is slightly larger than the previous estimate of $5500 \mathrm{~km} \mathrm{~s}^{-1}$. The blueshifted velocity change does not follow
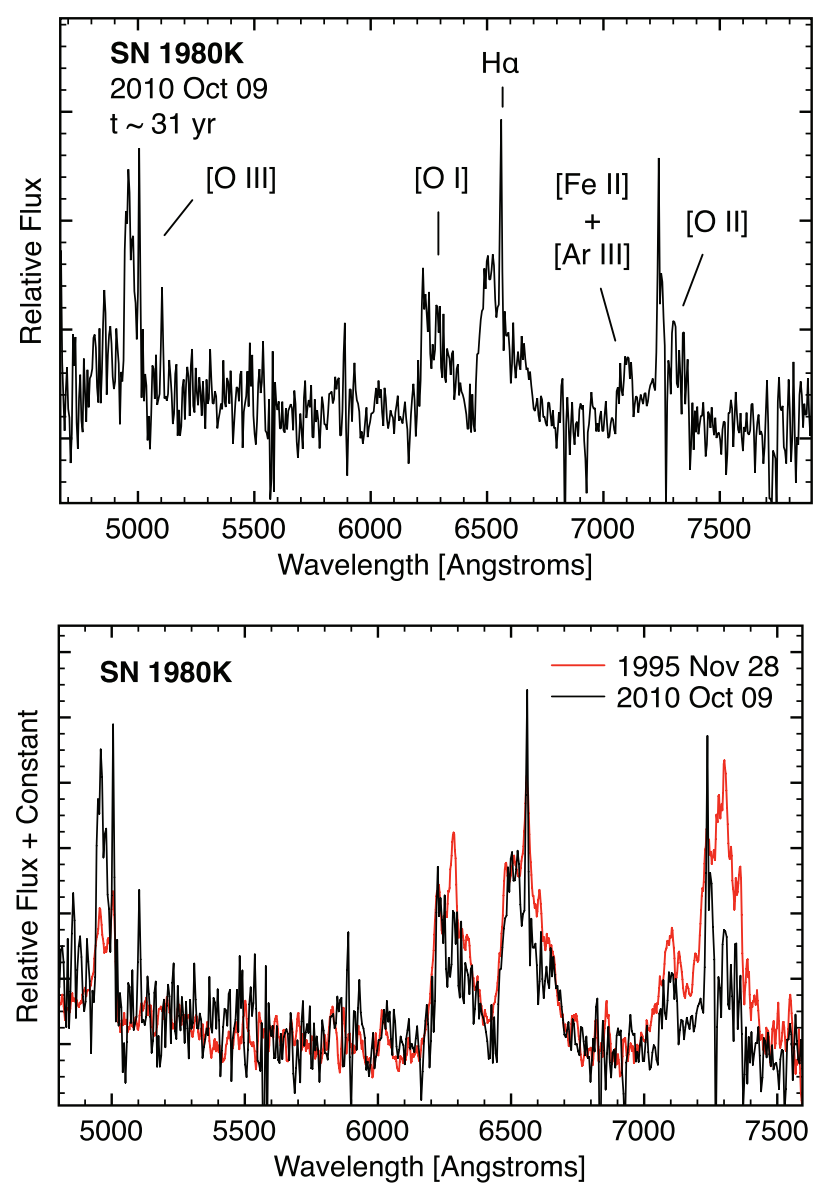

Figure 8. Top: MDM optical spectrum of SN 1980K 31 yr post-outburst. Bottom: the 2010 spectrum of SN $1980 \mathrm{~K}$ compared with a 1995 spectrum from Fesen et al. (1999).

(A color version of this figure is available in the online journal.)

the estimated rate of $\approx-400 \mathrm{~km} \mathrm{~s}^{-1} \mathrm{yr}^{-1}$ first noted by Fesen $\&$ Matonick (1994) and later confirmed by Fesen et al. (1995). [O I] has a velocity of $-4700 \mathrm{~km} \mathrm{~s}^{-1}$, which is considerably smaller than the $-6000 \mathrm{~km} \mathrm{~s}^{-1}$ reported in spectra from 1995. The substantial velocity change is likely associated with improved $\mathrm{S} / \mathrm{N}$.

We estimate a broad $\mathrm{H} \alpha$ flux of $(1.0 \pm 0.2) \times$ $10^{-15} \mathrm{erg} \mathrm{s}^{-1} \mathrm{~cm}^{-2}$, which is a small drop from $(1.3 \pm 0.2) \times$ $10^{-15} \mathrm{erg} \mathrm{s}^{-1} \mathrm{~cm}^{-2}$ reported by Fesen et al. (1999) $15 \mathrm{yr}$ earlier. 


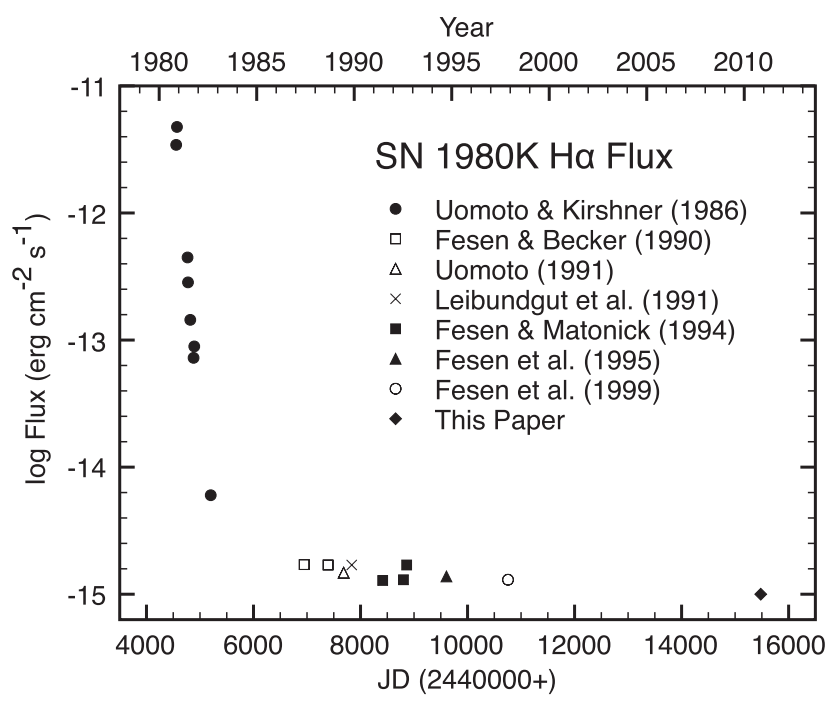

Figure 9. Observed H $\alpha$ flux in SN 1980K over the period 1980-2011.

In Figure 9, a plot of all published $\mathrm{H} \alpha$ flux measurements shows an almost flat emission trend over the past two decades. Persistent $\mathrm{H} \alpha$ emission with little to no decline over several years is consistent the other SN Type IIL already discussed, SN 1970G, and with the handful of additional Type IIL SNe observed years post-outburst including SN 1979C (Fesen \& Becker 1990; Fesen \& Matonick 1994; Fesen et al. 1995) and SN 1986E, whose brightness in $R$ at eight years of age was almost the same it was two years after outburst (Cappellaro et al. 1995). The origin of this emission has long been attributed to reverse shock-heated hydrogen-rich ejecta (e.g., Fesen et al. 1999; Milisavljevic et al. 2009; see Section 5) although Sugerman et al. (2012) suggest that SN 1980K's H $\alpha$ emission may have substantial contribution from scattered light echoes.

\subsection{SN 1993J}

SN 1993J in M81 ( $D \sim 3.6 \mathrm{Mpc}$; Freedman et al. 1994) was discovered by F. Garcia on 1993 March 28.9 (Ripero et al. 1993) and reached a maximum brightness of $V=10.8 \mathrm{mag}$ (Richmond et al. 1994). Early spectra showed an almost featureless blue continuum with indications of broad but weak $\mathrm{H} \alpha$ and $\mathrm{He}$ I $\lambda 5876$ suggesting a Type II event (Filippenko et al. 1993c; Garnavich \& Ann 1993). However, the spectra quickly evolved to show He I lines associated with the Type Ib class (Filippenko et al. 1993b), thus acquiring the transitional classification Type IIb (Filippenko et al. 1993a) anticipated by Woosley et al. (1987).

The relatively isolated location and nearby distance of SN 1993J has enabled close monitoring of emission in the X-ray (Zimmermann et al. 1994; Suzuki \& Nomoto 1995; Chandra et al. 2009), radio (Bartel et al. 1994, 2000; van Dyk et al. 1994; Weiler et al. 2007), and optical (Woosley et al. 1994; Filippenko et al. 1994; Matheson et al. 2000b, 2000a; Filippenko \& Matheson 2003; Fransson et al. 2005). Recently, photometric and spectroscopic observations a decade post-outburst detected the signature of a potential massive binary companion to the progenitor star (Maund et al. 2004; Maund \& Smartt 2009).

In the top panel of Figure 10, we present a 2009 December spectrum of SN 1993J. Identified emission lines have been marked, wavelengths have been corrected for a recession velocity of $-140 \mathrm{~km} \mathrm{~s}^{-1}$ (Matheson et al. 2000a), and a blue continuum has been removed using a third-order Chebyshev
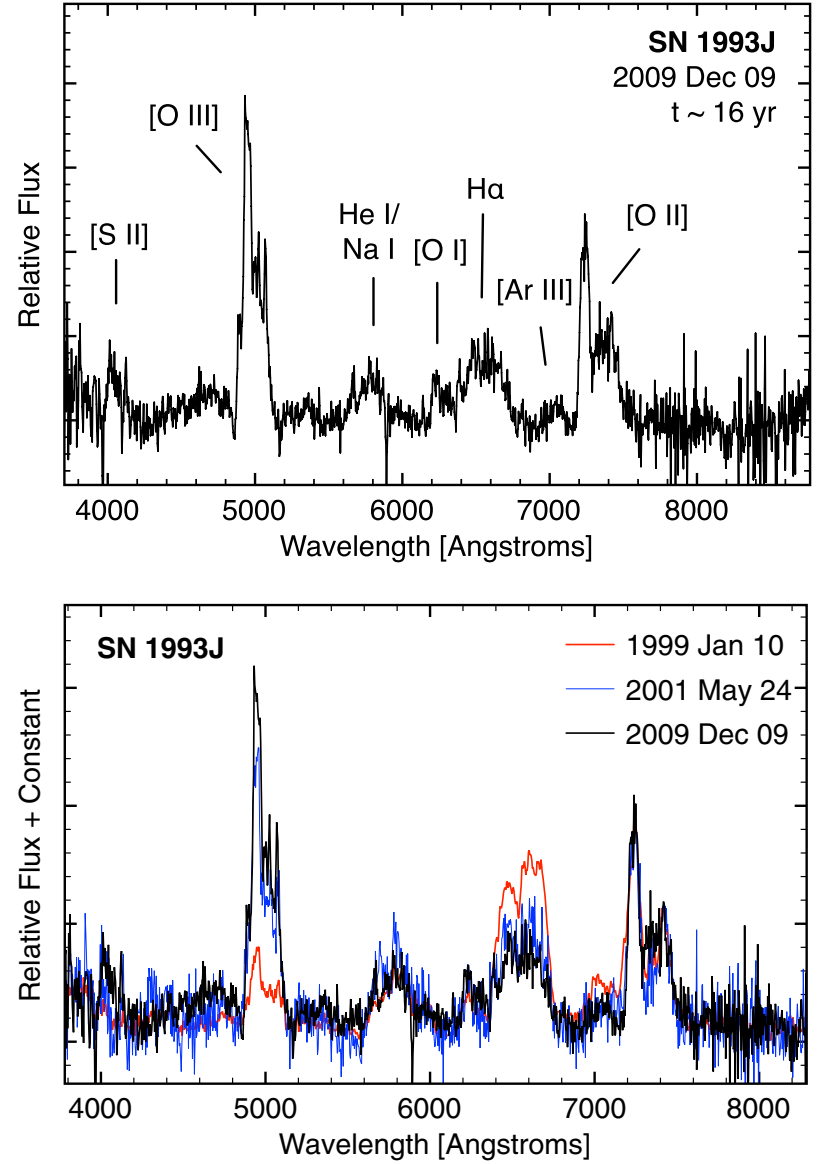

Figure 10. Top: MMT optical spectrum of SN 1993J 16 yr post-outburst with line identifications marked. Bottom: the 2009 spectrum of SN 1993J (above) compared with a 1999 spectrum from Matheson et al. (2000b, 2000a) and a previously unpublished 2001 spectrum.

(A color version of this figure is available in the online journal.)

function. In the lower panel of the figure, our 2009 spectrum is plotted over and directly contrasted with one obtained approximately $10 \mathrm{yr}$ earlier by Matheson et al. (2000b, 2000a) and a previously unpublished MMT spectrum obtained on 2001 May 24 kindly provided by T. Matheson and M. Modjaz. All spectra have been normalized to the [O II] emission lines to highlight the gradual increase in [O III] emission with respect to $\mathrm{H} \alpha$.

The general line profile shapes and small-scale features of the strongest emission lines have not changed significantly (see Figure 11). However, the ratio of $[\mathrm{O}$ III $] / \mathrm{H} \alpha$ emission has increased by over an order of magnitude, continuing a trend first noted by Matheson et al. (2000b). Previous spectra showed a persistent $\mathrm{H} \alpha$ line that, up until day 2452 after outburst, dominated the spectrum with an unusual, box-like profile with velocities in excess of $\pm 9000 \mathrm{~km} \mathrm{~s}^{-1}$. However, [O III] is now the dominant emission feature. The profile maintains its pronounced asymmetry toward blueshifted velocities with a major emission peak centered around $-3500 \mathrm{~km} \mathrm{~s}^{-1}$. We estimate a total [O III] $\lambda \lambda 4959,5007$ flux of $(9.5 \pm 0.2) \times 10^{-15} \mathrm{erg} \mathrm{s}^{-1} \mathrm{~cm}^{-2}$, implying a decline from the $1.6 \times 10^{-14} \mathrm{erg} \mathrm{s}^{-1} \mathrm{~cm}^{-2}$ measured in 1999 . The velocity span of -5800 to $+7000 \mathrm{~km} \mathrm{~s}^{-1}$ has remained unchanged.

The $[\mathrm{O}$ II $] \lambda \lambda 7319,7330$ lines follow the same profile distribution as [O $\mathrm{III}]$, as does [O I] with the exception that at around $6365 \AA$ it begins to merge with $\mathrm{H} \alpha$ (see Figure 11). The [S II] $\lambda \lambda 4069,4076$ lines have become more pronounced, exhibiting a blueshifted profile sharply peaked at $-3900 \mathrm{~km} \mathrm{~s}^{-1}$ and 

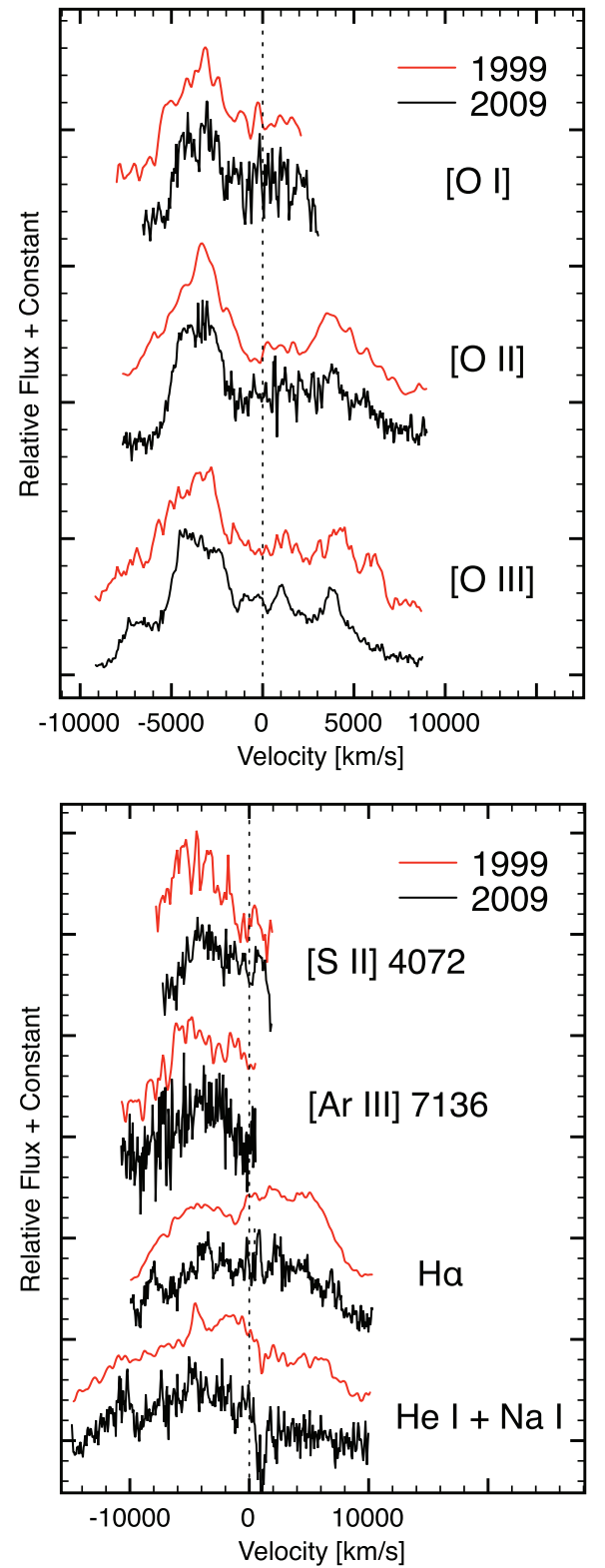

Figure 11. Emission line profiles in the spectra of SN 1993J in 1999 and 2009. In descending order, velocities are with respect to $6300,7325,5007,4072,7136$, 6563, and $5800 \AA$. The 1999 spectrum is from Matheson et al. (2000b, 2000a).

(A color version of this figure is available in the online journal.)

gradually sloping down to zero velocity. The blend of He I and $\mathrm{Na}$ I lines centered around $5800 \AA$ remains largely unchanged, though evidence for an emission peak centered around $5670 \AA$ has developed.

Emission around $7050 \AA$ has several possible sources of origin including $\mathrm{He}_{\mathrm{I}} \lambda 7065$, [Fe II] $\lambda 7155$, and [Ar III] $\lambda 7136$. Matheson et al. (2000b) attribute the feature to [Fe II $\lambda 7155$. However, we found that centering the velocity distribution with respect to $7136 \AA$ provided a good fit with the [S II] profile (see Figure 11). Thus, assuming a common origin for Ar and $\mathrm{S}$ from the $\mathrm{Si}-\mathrm{S}-\mathrm{Ar}-\mathrm{Ca}$ interior layer of the progenitor star, [Ar III] $\lambda 7136$ is likely the dominant contributor.

\subsection{Additional Late-time CCSN Spectra}

To help place the spectra of SN 1957D, 1970G, 1980K, and 1993J in context with other CCSNe observed at similarly evolved epochs, we retrieved archival late-time spectra for

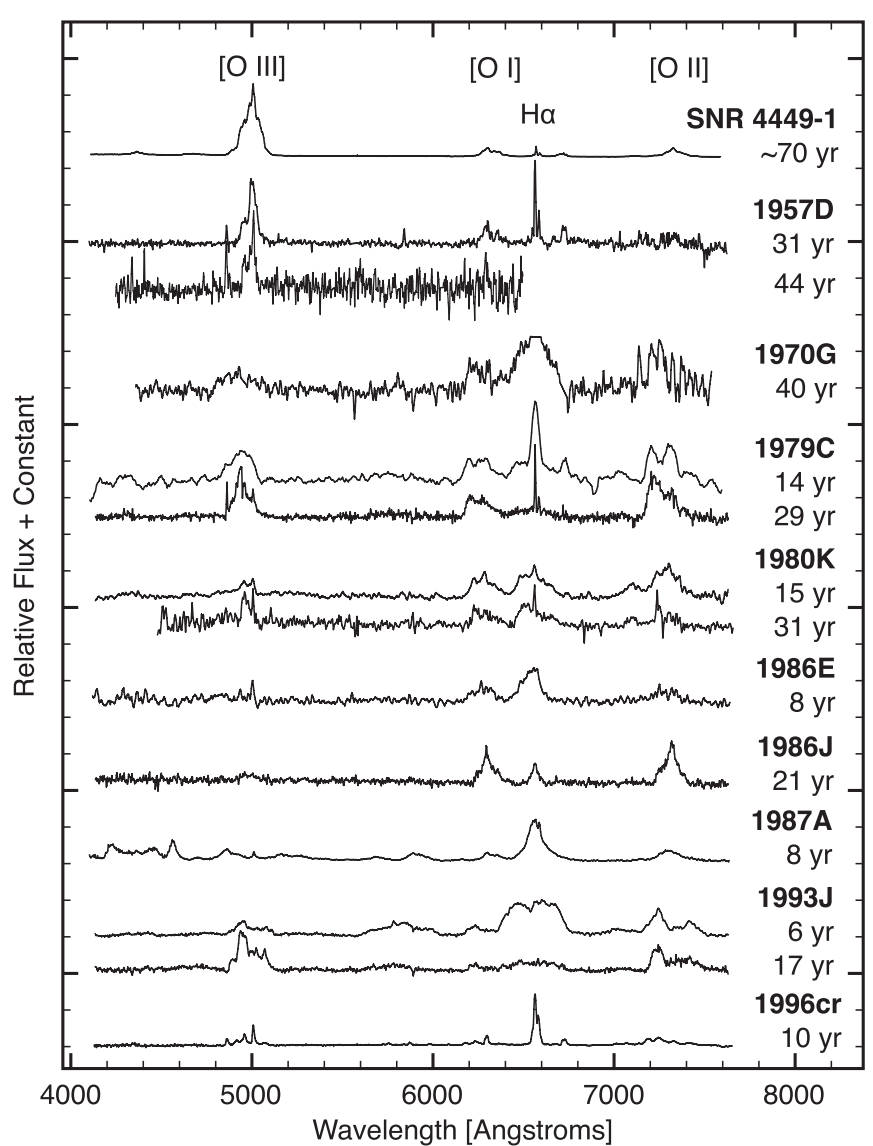

Figure 12. Optical spectra of intermediate-aged core-collapse supernovae exhibiting broad HWZI line widths $\gtrsim 2000 \mathrm{~km} \mathrm{~s}^{-1}$.

several CCSNe where broad (HWZI $\gtrsim 2000 \mathrm{~km} \mathrm{~s}^{-1}$ ) line emission associated with metal-rich ejecta was observed at epochs $\gtrsim 10$ yr. These objects include SN 1979C and SN 1986E (SN IIL), SN 1986J and SN 1996cr (SN IIn), and SN 1987A (SN IIpec).

Also included was the ultraluminous, O-rich SNR in NGC 4449, SNR 4449-1. SNR 4449-1 has no known classification, but properties of its late-time spectra and stellar environment are in line with a stripped-envelope progenitor (Seaquist \& Bignell 1978; Kirshner \& Blair 1980; Blair et al. 1983, 1984; Milisavljevic \& Fesen 2008). Recent work has suggested an age of $\sim 70 \mathrm{yr}$, which is comparable to the CCSNe studied here (Bietenholz et al. 2010). Spectra of all 10 SNe are shown in Figure 12 with their properties and references listed in Table 2.

Not included in our sample were some Type IIn CCSNe where strong Balmer emission associated with hydrogen-rich CSM dominates their spectra, e.g., SN 1978K (Ryder et al. 1993; Schlegel et al. 1999) and SN 1988Z (Aretxaga et al. 1999). Also not included was the unique event SN 1961V (Stringfellow et al. 1988) in light of its debated nature and limited optical spectra (Goodrich et al. 1989; Filippenko et al. 1995; Chu et al. 2004; Kochanek et al. 2011).

\section{DISCUSSION}

As can be seen from Figure 12, there is great variety in the latetime optical spectra of intermediate-aged CCSNe. Nonetheless, there exist some similarities between these objects with respect to changes in emission line strengths and widths over time. For example, the three SN IIL objects SN 1970G, 1979C, and 
Table 2

Details of Optical Spectra Presented in Figure 12

\begin{tabular}{lcccl}
\hline \hline SN & Type & Host Galaxy & Distance $(\mathrm{Mpc})^{\mathrm{a}}$ & \\
\hline SNR 4449-1 & $\ldots$ & NGC 4449 & $3.6^{*}$ & References \\
1957D & II & M83 & 4.6 & Cappellaro et al. (1995); this paper \\
1970G & IIL & M101 & 6.7 & This paper \\
1979C & IIL & M100 & 15.8 & Fesen et al. (1999); Milisavljevic et al. (2009) \\
1980K & IIL & NGC 6946 & 5.9 & Fesen et al. (1999); this paper \\
1986E & IIL & NGC 4302 & $24.9 *$ & Cappellaro et al. (1995) \\
1986J & IIn & NGC 891 & $10.2^{*}$ & Milisavljevic et al. (2008); Leibundgut et al. (1991) \\
1987A & IIpec & LMC & 0.05 & Chugai et al. (1997) \\
1993J & IIb & M81 & 3.7 & Matheson et al. (2000b, 2000a); this paper \\
1996cr & IIn & Circinus & $4.2^{*}$ & Bauer et al. (2008) \\
\hline
\end{tabular}

Notes.

${ }^{a}$ References to distance estimates provided in Sections 1 and 3 unless marked with an asterisk (*) in which case they are mean distances reported by Nasa/IPAC Extragalactic Database at http://ned.ipac.caltech.edu.

b Data retrieved from SUSPECT at http://bruford.nhn.ou.edu/ suspect/index 1.html.

c Data retrieved from MAST at http://archive.stsci.edu/.

1980K all show broad $\mathrm{H} \alpha\left(\simeq 5000 \mathrm{~km} \mathrm{~s}^{-1}\right)$ with the redshifted emission weaker than the blueshifted emission. These spectra also show comparable evolutionary changes in the strength of [O III] relative to $\mathrm{H} \alpha$. On the other hand, quite different late-time emissions are seen for objects such as SN 1986J and 1987A. Below we describe several properties that were found to be common to many of these spectra, and in Sections 5 and 6 we interpret their physical origin in the context of an SN-CSM interaction model and comparison to the young Galactic SNR Cassiopeia A.

\subsection{Changes in Relative Line Strength and Width Over Time}

In cases where multi-epoch spectra with good $\mathrm{S} / \mathrm{N}$ are available, the [O III] $\lambda \lambda 4959,5007$ and $\mathrm{H} \alpha$ lines change in strength relative to the $\left[\mathrm{O}_{\mathrm{I}}\right] \lambda \lambda 6300,6364$ and $\left[\mathrm{O}_{\mathrm{II}}\right] \lambda \lambda 7319$, 7330 lines. Namely, the flux ratio [O III $] /\left(\left[\mathrm{O}_{\mathrm{I}}\right]+[\mathrm{O}\right.$ II $]$ ) increases with time, and in most cases $\mathrm{H} \alpha /([\mathrm{O} \mathrm{I}]+[\mathrm{O} \mathrm{II}])$ decreases. This is clear in the spectra of three objects: SN $1980 \mathrm{~K}$ between 1995 and 2010 (see Figure 8), SN 1993J between 1999 and 2009 (see Figure 10), and in previously published spectra of SN 1979C between 1993 and 2008 (Milisavljevic et al. 2009). The fact that emission from the oldest SNe in our sample, SN 1957D and SNR $4449-1$, is dominated by [O III] emission with no appreciable $\mathrm{H} \alpha$ is also consistent with the trend.

However, some SNe remain dominated by $\mathrm{H} \alpha$ emission over all observed epochs. For instance, SN 1970G is dominated by broad $\mathrm{H} \alpha$ emission as it has been since first observations by Kirshner et al. (1973). Although we detected [O III] emission at $t=40 \mathrm{yr}$, its overall $\mathrm{H} \alpha$ strength is far stronger than other CCSNe of comparable ages. In the same category, SN 1986E at eight years of age continued to be $\mathrm{H} \alpha$-dominated with insignificant [O III] emission. The unique objects SN 1986J and SN 1987A also show weak or undetected [O III] emission.

A handful of SN spectra in our sample show measurable changes in the velocity widths of their emission line profiles. SN 1957D exhibited narrowing in its [O III] $\lambda \lambda 4959,5007$ lines of the order of $100 \mathrm{~km} \mathrm{~s}^{-1} \mathrm{yr}^{-1}$ (see Figure 3), a rate similar to changes in the velocity widths of forbidden oxygen emissions in SN 1979C between 1990 and 2008 (Milisavljevic et al. 2009). Narrowing of the order of $25 \mathrm{~km} \mathrm{~s}^{-1} \mathrm{yr}^{-1}$ was also noted in SN 1979C's $\mathrm{H} \alpha$ emission, but only affecting blueshifted velocities. In some cases no velocity changes were measured (e.g., SN 1993J), while in other cases changes in velocity width could not be measured confidently owing to poor $\mathrm{S} / \mathrm{N}$ and differences in detector sensitivity and/or spectral resolution (e.g., SN 1970G).

Changes in velocity widths of emission line profiles are predicted to be good tests between various mechanisms of late-time emission (Chevalier \& Fransson 1992, 1994). In circumstellar interaction scenarios where the reverse shock penetrates into deeper layers of ejecta, the velocity widths of emission lines are anticipated to narrow. Alternatively, scenarios involving a pulsar wind nebula where emission is powered by photoionization, line widths are anticipated to broaden because of acceleration by the pulsar bubble. With one exception, all $\mathrm{SNe}$ studied here show either no discernible change in their line widths or experienced narrowing of the order of $\sim 100 \mathrm{~km} \mathrm{~s}^{-1} \mathrm{yr}^{-1}$.

\subsection{Blueshifted Line Profiles}

Blueshifted line profiles are quite common in the latetime CCSN spectra. Emission asymmetry is strongest in the forbidden oxygen lines, particularly [O III], but is also observed in $\mathrm{H} \alpha$ line profiles where it is generally less pronounced. The oxygen line profiles also show conspicuous emission line substructure.

The presence of blueshifts in many late-time CCSN spectra is illustrated in the top panel of Figure 13, where we show line profiles of [O III] emission for SN 1979C, 1980K, 1993J, and $1996 \mathrm{cr}$. These SNe exhibit noticeably asymmetric profiles dominated by blueshifted emission with velocities in excess of $-3000 \mathrm{~km} \mathrm{~s}^{-1}$. SN $1979 \mathrm{C}$ and $1980 \mathrm{~K}$, showing very little redshifted emission, are extreme examples of this phenomenon. In the case of SN 1996cr, evidence for two blueshifted peaks can be seen. The blueshifted asymmetry and line substructure is also present in [O II] line emission, which is shown in the bottom panel of Figure 13.

These asymmetric oxygen emission line profiles suggest that internal absorption is obscuring emission from the SN's receding rear hemisphere. One candidate for this type of absorption at late epochs is dust formation in metal-rich ejecta much like that seen in SN 1987A (see McCray 1993 and references therein). Beginning around 450 days after optical maximum, SN 1987A exhibited an increase in infrared emission accompanied shortly thereafter with a noticeable blueshift in the $[\mathrm{MgI}],\left[\mathrm{O}_{\mathrm{I}}\right]$, and $\left[\mathrm{C}_{\mathrm{I}}\right]$ emission lines. The blueshift was attributed to an 

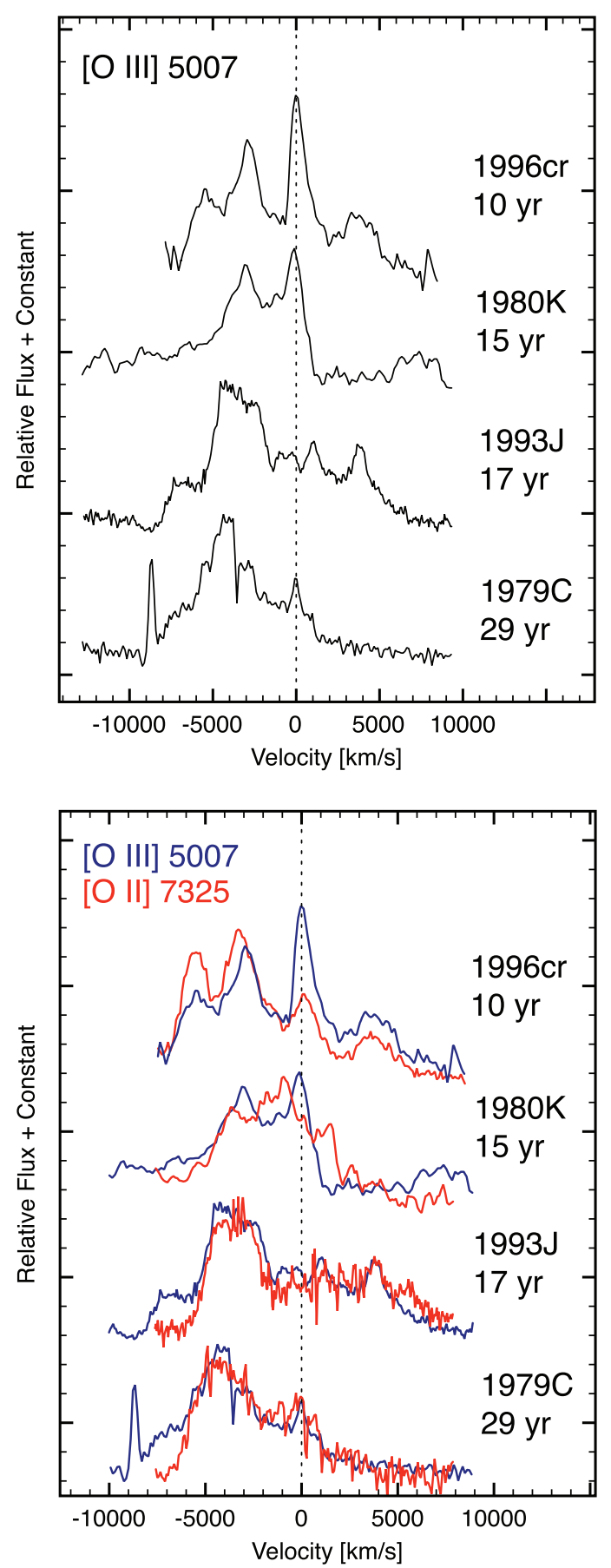

Figure 13. Blueshifted emission line profiles from spectra presented in Figure 12. Top: [O III] $\lambda \lambda 4959,5007$ line profiles. Velocities are with respect to $5007 \AA$ A. Bottom: [O III] profiles compared to [O II] $\lambda \lambda 7319,7330$ profiles from the same epoch. Velocities are with respect to $7325 \AA$.

(A color version of this figure is available in the online journal.)

attenuation of emission from the receding side of the ejecta by dust grains formed within the ejecta, and the infrared excess associated with the subsequent re-radiation by the dust grains.

Absorption due to dust in SN ejecta has also been suggested to explain the late-time asymmetric UV line profiles observed in SN 1979C (Fesen et al. 1999). Double-peaked profiles with the blueward peak substantially stronger than the red were seen in C II] $\lambda \lambda 2324,2325$, [O II] $\lambda \lambda 2470,2470$, and $\mathrm{Mg}$ II $\lambda \lambda 2796,2803$ emissions. A handful of additional cases where dust formation in ejecta has been proposed include SN 1990I
(Elmhamdi et al. 2004), 1999em (Elmhamdi et al. 2003), 2003gd (Sugerman et al. 2006; Meikle et al. 2007), 2005af (Kotak et al. 2006), 2007it (Andrews et al. 2011), and 2007od, where it may have had a clumpy structure (Inserra et al. 2011; Andrews et al. 2010).

Alternatively, absorption by dust may take place in the cool dense shell (CDS) in the shock region (Deneault et al. 2003). This is a likely place for dust formation because of its high density and low temperature (Fransson 1984; Chevalier \& Fransson 1994). Assuming that the CDS is formed behind the reverse shock (in the Lagrangian reference frame), both the front- and back-side line emissions will be absorbed by the dust, though hydrodynamic mixing giving rise to a clumpy CDS can allow some emission to penetrate (Chevalier \& Blondin 1995). Formation of dust in a CDS has been reported in at least three supernovae within months of outburst including $1998 \mathrm{~S}$ (Gerardy et al. 2000), 2005ip (Fox et al. 2009; Smith et al. 2009), and 2006jc (Smith et al. 2008). It was also reported in SN 2004et, which exhibited a wide $\pm 8500 \mathrm{~km} \mathrm{~s}^{-1}$ box-shaped $\mathrm{H} \alpha$ emission about two years past outburst with a blueshifted profile resembling many of the oxygen profiles presented here (Kotak et al. 2009; see Figure 4 of that paper).

However, it is important to note that not all supernovae in our sample show large and pronounced blueshifted oxygen or hydrogen line emission. SN 1957D, 1986J, 1987A, and 1986E only exhibit relatively small velocity blueshifts of approximately $-500 \mathrm{~km} \mathrm{~s}^{-1}$. Another exception is the ultraluminous oxygen-rich supernova remnant SNR 4449-1. Unlike the other supernovae of this group, pronounced asymmetry is seen in SNR 4449-1's [S II] $\lambda \lambda 6716,6731$ and [Ar III] $\lambda 7136$ emission profiles which exhibit blueshifted velocity distributions spanning $-2500<V_{\exp }<500 \mathrm{~km} \mathrm{~s}^{-1}$ (Milisavljevic \& Fesen 2008; Bietenholz et al. 2010).

\section{MODELS OF SN-CSM INTERACTION}

The optical emissions from the supernovae discussed here are likely to be from circumstellar interaction, except for SN 1987A where radioactivity is a plausible power source at an age of eight years (Chugai et al. 1997; Kozma \& Fransson 1998a, 1998b; Larsson et al. 2011). Thus, the changes in relative line strengths and widths of the $\mathrm{O}$ and $\mathrm{H}$ lines noted in Section 3 are useful tests of SN-CSM interaction models.

If the supernova and circumstellar gas is evenly distributed, the interaction is expected to lead to brighter optical emission from the reverse shock region as opposed to the forward shock because of the higher density there (Chevalier \& Fransson 1994, 2003). The forward shock generally is nonradiative, but radiative shocks can occur in this region if the circumstellar gas is very clumpy. This is the likely source of the narrow lines in Type IIn supernovae (Chugai \& Danziger 1994).

Supernovae of Type IIL and IIb show broader optical line emission and are plausibly described by the scenario of reverse and forward shocks. Estimates of the mass-loss density for these events can be obtained from radio observations (Weiler et al. 2002; Montes et al. 1997) and are shown in Table 3, where $\dot{M}_{-5}$ is the progenitor mass loss rate in units of $10^{-5} M_{\odot} \mathrm{yr}^{-1}$ and $v_{w 1}$ is the wind velocity in units of $10 \mathrm{~km} \mathrm{~s}^{-1}$. Several assumptions are made in obtaining these estimates; in particular, the preshock wind temperature is assumed to be $2 \times 10^{4} \mathrm{~K}$ and the shock velocity is $10^{4} \mathrm{~km} \mathrm{~s}^{-1}$. The value for SN $1986 \mathrm{E}$ is from Montes et al. (1997) and is scaled to the lower limit values of Weiler et al. (2002) on SN 1979C and SN 1980K. Overall, the wind 
Table 3

Shocked Ejecta Masses at the Epochs of Spectra

\begin{tabular}{lccccc}
\hline \hline SN & Type & $\dot{M}_{-5} / v_{w 1}$ & $\begin{array}{c}\text { Age } \\
(\mathrm{yr})\end{array}$ & $\begin{array}{c}M_{e j} / V_{4} \\
\left(M_{\odot}\right)\end{array}$ & H line \\
\hline $1993 \mathrm{~J}$ & IIb & 2.4 & 6 & 0.1 & Present \\
$1993 \mathrm{~J}$ & IIb & 2.4 & 17 & 0.3 & Weak \\
$1970 \mathrm{G}$ & IIL & 6.8 & 40 & 2.2 & Present \\
$1979 \mathrm{C}$ & IIL & 11 & 14 & 1.3 & Present \\
$1979 \mathrm{C}$ & IIL & 11 & 29 & 2.6 & Weak \\
$1980 \mathrm{~K}$ & IIL & 1.3 & 31 & 0.3 & Present \\
$1986 \mathrm{E}$ & IIL & 3 & 8 & 0.2 & Present \\
\hline
\end{tabular}

densities are at the high end of wind densities estimated for red supergiant stars.

In the initial phase of interaction, the gas heated at the reverse shock radiatively cools and there is optical emission from both the cooling shock wave and preshock ejecta that have been radiatively heated (Chevalier \& Fransson 1994, 2003). During this phase, the shock luminosity drops approximately as $v_{\mathrm{sh}}^{3}$, where $v_{\text {sh }}$ is the forward shock velocity. The transition to a noncooling phase occurs at

$$
\begin{aligned}
t_{\mathrm{cr}}= & 0.0017(n-3)(n-4)(n-2)^{3.34} \\
& \times\left(\frac{V_{\mathrm{ej}}}{10^{4} \mathrm{~km} \mathrm{~s}^{-1}}\right)^{-5.34}\left(\frac{\dot{M}_{-5}}{v_{w 1}}\right) \text { day, }
\end{aligned}
$$

where $n$ is the power-law exponent of the ejecta density profile and $V_{\text {ej }}$ is the highest velocity in the freely expanding ejecta. It can be seen that the result is very sensitive to $n$ (expected to be in the range 7-12) and the velocity. By this estimate, the cases considered here are expected to be in the nonradiative phase, although Nymark et al. (2009) model SN 1993J as being in the radiative phase at an age of just eight years.

Another factor is the composition of matter at the reverse shock. Equation (1) assumes solar abundances and an enhancement of heavy elements prolongs the radiative phase. The optical emission is expected to be mainly from the pre-reverse shock ejecta. Some emission from the CDS built up during the radiative phase is possible, but that gas is expected to disperse with time.

This model allows an estimate of the circumstellar gas that has been swept up by the forward shock front:

$$
M_{\mathrm{cs}}=\frac{\dot{M}}{v_{w}} V t=0.01 \dot{M}_{-5} v_{w 1}^{-1} V_{4} t_{\mathrm{yr}} M_{\odot},
$$

where $V$ is the average velocity of the forward shock and $V_{4}$ is in units of $10^{4} \mathrm{~km} \mathrm{~s}^{-1}$. The mass of shocked ejecta, $M_{\mathrm{ej}}$, is simply related to $M_{\mathrm{cs}}$ if the supernova density profile is a power law with index $n$ (Chevalier 1982). For $n=7$, we have $M_{\mathrm{ej}} / M_{\mathrm{cs}}=0.82$, which is the value used in Table 3 . This value of $n$ is smaller than the high value that might be expected at the outer part of the supernova because the reverse shock has moved into the supernova at these late times. The mass ratio is a factor $\sim 2$ smaller for $n=6$ and the same factor larger for $n=9$.

Within these uncertainties, the results in Table 3 are consistent with the view that the decline of $\mathrm{H}$ emission in these supernovae, where it occurs, is due to all of the $\mathrm{H}$ envelope having passed through the reverse shock wave. In the case of SN 1993J, models of the early supernova indicate an $\mathrm{H}$ envelope mass of $\sim 0.2 \mathrm{M}_{\odot}$ (Woosley et al. 1994). This mass would have been shocked by the time of the observation at $t=17 \mathrm{yr}$, so there is no longer $\mathrm{H}$ present at the reverse shock. Type IIL supernovae are believed

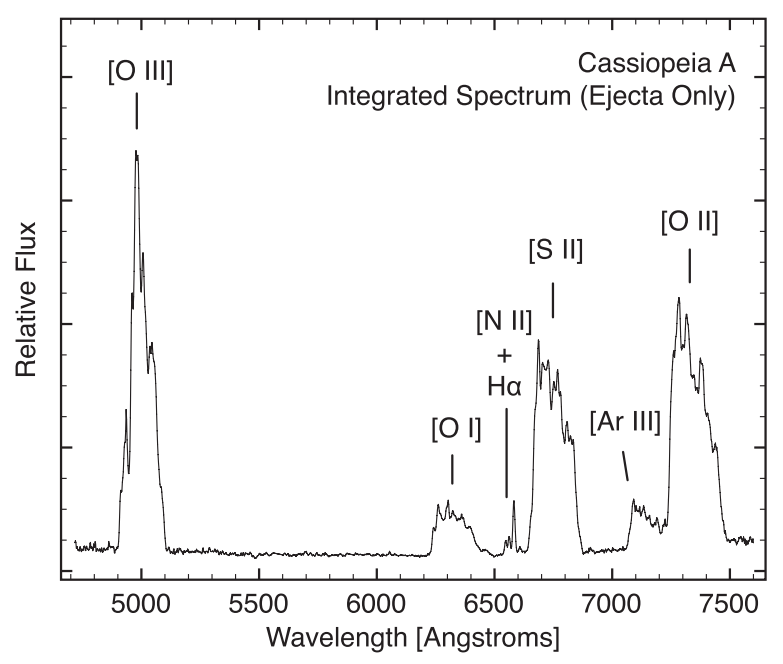

Figure 14. Spatially integrated spectrum of the main shell of the Cassiopeia A supernova remnant.

to have higher $\mathrm{H}$ envelope masses than Type $\mathrm{IIb}$ events. For instance, Blinnikov \& Bartunov (1993) find that $\mathrm{H}$ envelopes with mass $\sim 1-2 M_{\odot}$ are present. Thus, the disappearance of the broad H emission in SN 1979C is consistent within this framework. The observations of the $\mathrm{H}$ line compared to $\mathrm{O}$ lines are generally in accord with expectations, and there is the prediction that the $\mathrm{H}$ line in SN 1970G should fade in the not-too-distant future.

Another expectation of the circumstellar interaction scenario is that the interaction leads to the deceleration of the gas near the reverse shock front. For the power-law density profile, we have a radial expansion $R \propto t^{m}$, where $m=(n-3) /(n-2)$ (Chevalier 1982). The highest velocity at the reverse shock evolves as

$$
\begin{aligned}
\frac{d V_{\mathrm{ej}}}{d t}= & -(1-m) \frac{V_{\mathrm{ej}}}{t}=-100\left(\frac{1-m}{0.2}\right) \\
& \times\left(\frac{V_{\mathrm{ej}}}{10^{4} \mathrm{~km} \mathrm{~s}^{-1}}\right)\left(\frac{t}{20 \mathrm{yr}}\right)^{-1} \mathrm{~km} \mathrm{~s}^{-1} \mathrm{yr}^{-1},
\end{aligned}
$$

where $n=7$ is used for the reference value of $m$. The expected rate of line narrowing is close to that observed.

\section{CCSNe AND SNRs: COMPARISONS TO Cas A}

In an effort to understand the nature of blueshifted and multiple peak emission line profiles and relate their features with kinematic properties of the optically emitting ejecta, a spatially integrated spectrum of the young Galactic SNR Cassiopeia A (Cas A) was constructed. Cas A is the prototype for the class of young, oxygen-rich SNRs and provides a clear look at the explosion dynamics of a CCSN (van den Bergh 1988; Fesen 2001). Recent detection of optical echoes of the supernova outburst indicates that it was a Type IIb event, probably from a red supergiant progenitor with mass $10-30 M_{\odot}$ that may have lost much of its hydrogen envelope to a binary interaction (Krause et al. 2008; Rest et al. 2011; Fabian et al. 1980; Vink et al. 1996; Young et al. 2006). A nearby distance of $3.4 \mathrm{kpc}$ has permitted detailed study of Cas A's composition and distribution of supernova ejecta on fine scales (Reed et al. 1995; Fesen et al. 2001 ), and its estimated current age of $\approx 330 \mathrm{yr}$ places it at a stage of evolution not that different from the intermediate-aged CCSNe of our sample (Thorstensen et al. 2001; Fesen et al. 2006). 


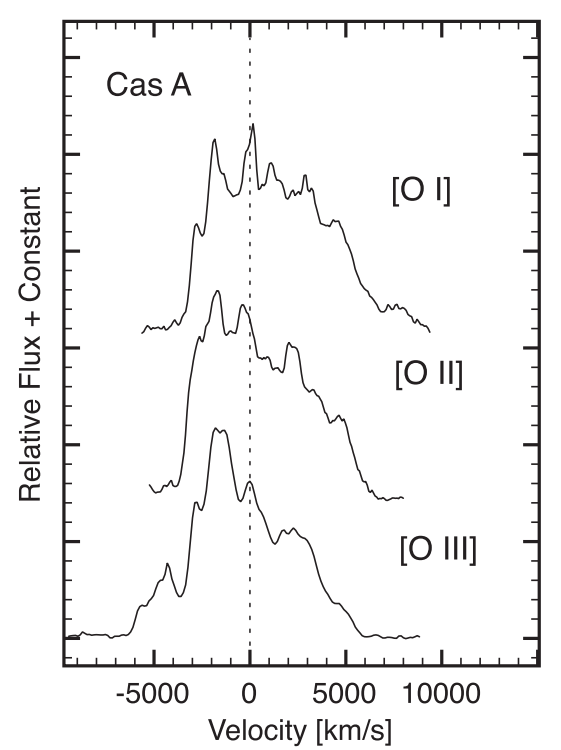

Figure 15. [O I] $\lambda \lambda 6300,6364,\left[\mathrm{O}_{\mathrm{II}}\right] \lambda \lambda 7319,7330$, and [O III] $\lambda \lambda 4959,5007$ line profiles from the integrated spectrum of Cassiopeia A.

Our integrated spectrum of the Cas A SNR is presented in Figure 14. The spectrum was extracted from 80 long slit spectra spaced 3 " apart across the entire main shell (approximately $4^{\prime}$ in diameter) covering the wavelength region $4500-8000 \AA$ (resolution $7 \AA$ ) from an investigation of the remnant's threedimensional kinematic structure (D. Milisavljevic \& R. A. Fesen 2012 , in preparation).

This spectrum has not been corrected for reddening which can vary $4 \lesssim A_{V} \lesssim 8$ (Hurford \& Fesen 1996), so [O III] emission is intrinsically much more dominant than the presented spectrum indicates. These spectra also largely omit emission from the remnant's slow-moving CSM called quasi-stationary flocculi. Additionally, the spectrum does not include emission from the faint NE jet or other outer ejecta which contribute relatively insignificant optical flux.

\subsection{Cas A's Asymmetric Emission Profiles}

The Cas A spectrum (Figure 14) shows pronounced blueshifted emission with conspicuous line substructure in [O I], [O II], [O III], [S II], and [Ar III]. These line profiles are all asymmetric, with bulk blueshifts peaked around $-1700 \mathrm{~km} \mathrm{~s}^{-1}$ and a blue to red asymmetry that increases somewhat with higher ionization levels. The velocity line profiles of the forbidden oxygen lines are shown in Figure 15. The [S II] $\lambda \lambda 6716,6731$ and [Ar III] $\lambda 7136$ lines, not shown in Figure 15, have comparable blueshifts of around $-1700 \mathrm{~km} \mathrm{~s}^{-1}$.

The Cas A spectrum appears similar to many of the intermediate-aged CCSNe exhibiting strong [O III] emission. Particularly well matched with Cas A are SN 1979C, SN 1993J, SNR 4449-1, and to some degree SN 1980K (see Figure 13), which show strong blueshifted oxygen emissions and/or conspicuous line substructure.

In Figure 16, the [O III] emission line profile of the integrated Cas A spectrum is plotted along with the profiles of SN 1993J and SNR 4449-1 to highlight their similar spectral features. It is interesting to note that Cas A and SN 1993J were both Type IIb $\mathrm{SNe}$ at photospheric stages, and here we see that they share late-time spectral features as well.

Extensive multi-wavelength studies of Cas A allow strong ties to be made between the emission asymmetry and absorption due

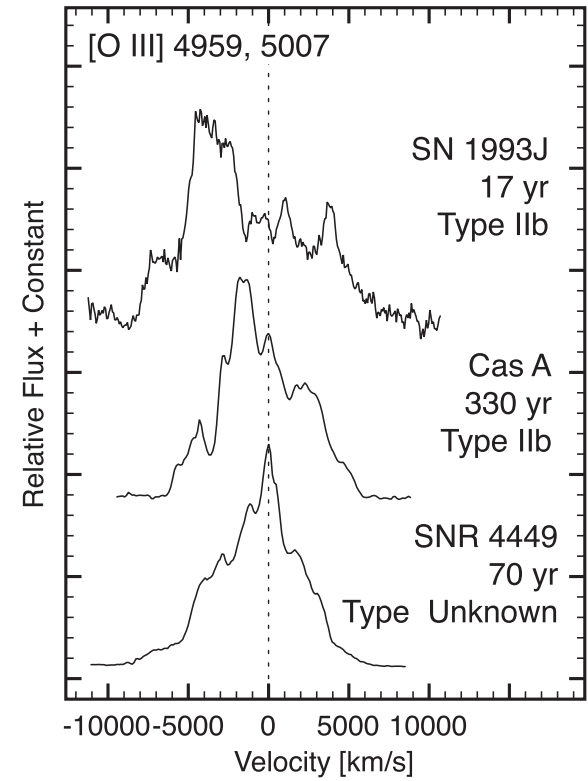

Figure 16. [O III] $\lambda \lambda 4959,5007$ emission line profiles of SN 1993J, SNR 44491, and the integrated Cas A spectrum. Velocities are with respect to $5007 \AA$. Conspicuous line substructure is seen in all three profiles.

to dust in the ejecta. Cas A is the only Galactic SNR that exhibits clear evidence of dust formed in its ejecta (Lagage et al. 1996; Arendt et al. 1999; Dwek 2004; Krause et al. 2004). Estimates of the total mass remain controversial, ranging over several orders of magnitude from less than $3 \times 10^{-3} M_{\odot}$ to over $4 M_{\odot}$. However, most recently Spitzer infrared observations showed close overlap between the ejecta and dust maps of the remnant indicating freshly formed dust in the ejecta having a total dust mass in the range of $0.020-0.054 M_{\odot}$ (Rho et al. 2008).

\subsection{Ejecta Rings: Are They Common?}

The three-dimensional kinematic properties of Cas A's ejecta have been studied in considerable detail (Lawrence et al. 1995; Reed et al. 1995; DeLaney et al. 2010; D. Milisavljevic \& R. A. Fesen 2012, in preparation). In general, Cas A's optical emission takes the form of an approximately spherical shell made up of several large ring-like structures composed of O- and S-rich material with radii of $\sim 1^{\prime}(\sim 1 \mathrm{pc})$. Its brightest optical emission comes from two continuous rings of material along its northern limb which are distinct in velocity space: a larger ring of highly redshifted material spanning radial velocities +5500 to $0 \mathrm{~km} \mathrm{~s}^{-1}$ and a smaller ring of mostly blueshifted material spanning 0 to $-2500 \mathrm{~km} \mathrm{~s}^{-1}$. A handful of additional broken and complete rings contribute to a velocity asymmetry in the radial velocities of order -4500 to $6000 \mathrm{~km} \mathrm{~s}^{-1}$.

The observed line profiles of Cas A's integrated spectrum indicate that internal absorption obscures or "hides" important kinematic properties of its ejecta. Given that the integrated profile resulting from a ring or torus of material suffering no opacity effects is double-peaked (such that each individual peak is centered at the approaching and receding velocity edges of the ring), a collection of co-added double-peaked profiles would be expected from the many ejecta rings that make up the remnant. Instead, these ejecta rings manifest themselves in emission line profiles as line substructures superimposed on an overall blueshifted line emission. Thus, the combination of internal absorption with the wide range of orientations, velocities, and discontinuities of the ejecta rings produces an 
integrated emission profile not indicative of its multi-ringed nature.

Emission line substructure in late-time spectra of CCSNe like that observed in SN 1993J and SNR 4449-1 (Figure 16) is typically identified as "blobs" or "clumps" of ejecta (Matheson et al. 2000b; Milisavljevic \& Fesen 2008). However, the multipeaked line substructure seen in Cas A's emissions originates from its multi-ringed distribution of ejecta. Hence, the similarity between the emission line profiles of the Cas A SNR and decades-old CCSNe raises the possibility that the long-lived emission line substructure of these extragalactic events may also be linked with SN debris arranged in large-scale rings being excited by a reverse shock like that observed in Cas A.

\section{CONCLUSIONS}

Optical HST images and ground-based spectra of several CCSNe obtained years to decades after outburst were investigated in an effort to understand some of their late-time optical emission properties. New observations of SN 1957D, 1970G, $1980 \mathrm{~K}$, and 1993J were presented, examined, and compared to archival late-time spectra of six additional CCSNe retrieved from the literature (SN 1979C, 1986E, 1986J, 1987A, 1996cr, and SNR 4449-1). Temporal evolution in the relative strengths and profiles of strong emission lines were inspected with particular attention to hydrogen and oxygen emissions.

Several common properties observed in the late-time optical spectra were consistent with expectations for emissions produced by interaction between the $\mathrm{SN}$ ejecta and the progenitor star's CSM. These properties include conspicuously declining $\mathrm{H} \alpha /\left(\left[\mathrm{O}_{\mathrm{I}}+\left[\mathrm{O}_{\mathrm{II}}\right]\right)\right.$ and increasing $[\mathrm{O} \mathrm{III}] /([\mathrm{O} \mathrm{I}]+[\mathrm{O} \mathrm{II}])$ flux ratios over time, as well as narrowing velocity widths of emission lines. The decline of $\mathrm{H}$ emission is most likely due to the $\mathrm{H}$ envelope having passed through the reverse shock wave, and the line narrowing the result of the deceleration of the interaction shell between the SN ejecta and the CSM at a rate of $\sim 100 \mathrm{~km} \mathrm{~s}^{-1} \mathrm{yr}^{-1}$.

Asymmetric emission line profiles in oxygen and/or hydrogen emissions with one or more blueshifted emission peaks were also found to be a common and long-lasting phenomenon. All spectra in our sample (see Figure 12) exhibit emission profiles having bulk blueshifts - but never redshifts - suggestive of dust formation within the ejecta or the CDS in the shock region. Many spectra also show conspicuous line substructure across ionization species.

To further investigate the nature of these emission line asymmetries and substructures, an integrated optical spectrum of the 330 yr old Galactic SNR Cas A was created to simulate what a CCSN remnant would look like as an unresolved extragalactic source. Blueshifted profiles with extensive line substructure were seen in the most prominent lines of [O I ], [O II], [O III], $[\mathrm{S}$ II $]$, and [Ar III]. The kinematic properties of the ejecta were mapped with these line profiles. The multi-peaked line profiles were associated with previously identified large ring-like structures of high-velocity ejecta, and the lines' blueshifted velocities were linked to internal absorption resulting from dust known to reside within the ejecta.

Remarkable similarities are seen between the Cas A spectrum and the intermediate-aged CCSN spectra of our sample. The correspondence of late-time spectral features between Cas A and SN 1993J is particularly strong, which is interesting given that both were Type IIb events. The shared spectral features are consistent with the view that emission line asymmetry observed in many evolved CCSN spectra may be associated with dust in the ejecta. Furthermore, the similarities between these supernovae suggest that emission line substructures typically interpreted as ejecta "clumps" or "blobs" in intermediate-aged CCSNe may actually be linked with large-scale rings of ejecta as seen in Cas A. It is worthwhile to note that Cas A is not unique in the spatial distribution of its metal-rich ejecta, as other young SNRs such as 1E 0102-7219 (Tuohy \& Dopita 1983; Eriksen et al. 2001; Vogt \& Dopita 2010) and N132D (Morse et al. 1995; Vogt \& Dopita 2011) also exhibit ejecta arranged in ring-like geometries.

We thank the referee for comments and suggestions that improved the manuscript. We also thank John Thorstensen and MDM staff for help with telescope operations, particularly with configuring the new acquisition camera which made some of these observations possible, T. Matheson and M. Modjaz for making the 2001 spectrum of SN 1993J available, and F. Bauer for providing a spectrum of SN 1996cr. D.M. and R.A.F. acknowledge support by NSF through grant AST-0908237. R.A.C. acknowledges support from NSF grant AST-0807727. M.T. is supported by the PRIN-INAF 2009 "Supernovae Variety and Nucleosynthesis Yields" and by the grant ASI-INAF $\mathrm{I} / 009 / 10 / 0$. This work is based in part on observations made with the NASA/ESA Hubble Space Telescope, and obtained from the Hubble Legacy Archive, which is a collaboration between the Space Telescope Science Institute (STScI/NASA), the Space Telescope European Coordinating Facility (ST-ECF/ESA), and the Canadian Astronomy Data Centre (CADC/NRC/CSA). Some data presented here were obtained at the MMT Observatory, a joint facility of the Smithsonian Institution and the University of Arizona. Supernova research at the Harvard College Observatory is supported by the National Science Foundation through grants AST-0606772 and AST-0907903. This research has made use of SAOImage DS9, developed by Smithsonian Astrophysical Observatory, and used the facilities of the Canadian Astronomy Data Centre operated by the National Research Council of Canada with the support of the Canadian Space Agency.

\section{REFERENCES}

Allen, R. J., Goss, W. M., Ekers, R. D., \& de Bruyn, A. G. 1976, A\&A, 48, 253 Andrews, J. E., Gallagher, J. S., Clayton, G. C., et al. 2010, ApJ, 715, 541 Andrews, J. E., Sugerman, B. E. K., Clayton, Geoffrey, C., et al. 2011, ApJ, 731,47

Arendt, R. G., Dwek, E., \& Moseley, S. H. 1999, ApJ, 521, 234

Aretxaga, I., Benetti, S., Terlevich, R. J., et al. 1999, MNRAS, 309, 343

Barbon, R., Ciatti, F., \& Rosino, L. 1979, A\&A, 72, 287

Barbon, R., Ciatti, F., \& Rosino, L. 1982, A\&A, 116, 35

Bartel, N., Bietenholz, M. F., Rupen, M. P., et al. 1994, Nature, 368, 610 Bartel, N., Bietenholz, M. F., Rupen, M. P., et al. 2000, Science, 287, 112 Bauer, F. E., Dwarkadas, V. V., Brandt, W. N., et al. 2008, ApJ, 688, 1210 Bietenholz, M. F., Bartel, N., Milisavljevic, D., et al. 2010, MNRAS, 409, 1594 Blair, W. P., Kirshner, R. P., \& Winkler, P. F., Jr. 1983, ApJ, 272, 84

Blair, W. P., Raymond, J. C., Gull, T. R., \& Fesen, R. A. 1984, ApJ, 279, 708 Blinnikov, S. I., \& Bartunov, O. S. 1993, A\&A, 273, 106

Brown, R. L., \& Marscher, A. P. 1978, ApJ, 220, 467

Buta, R. J. 1982, PASP, 94, 578

Canizares, C. R., Kriss, G. A., \& Feigelson, E. D. 1982, ApJ, 253, L17

Cappellaro, E., Danziger, I. J., \& Turatto, M. 1995, MNRAS, 277, 106

Chandra, P., Dwarkadas, V. V., Ray, A., Immler, S., \& Pooley, D. 2009, ApJ, 699, 388

Chevalier, R., \& Blondin, J. M. 1995, ApJ, 444, 312

Chevalier, R. A. 1982, ApJ, 258, 790

Chevalier, R. A., \& Fransson, C. 1992, ApJ, 395, 540

Chevalier, R. A., \& Fransson, C. 1994, ApJ, 420, 268

Chevalier, R. A., \& Fransson, C. 2003, in Supernovae and Gamma-Ray Bursters, ed. K. Weiler (Lecture Notes in Physics, Vol. 598; Berlin: Springer), 171 
Chevalier, R. A., \& Fransson, C. 2006, ApJ, 651, 381

Chu, Y., Gruendl, R. A., Stockdale, C. J., et al. 2004, AJ, 127, 2850

Chugai, N. N., Chevalier, R. A., Kirshner, R. P., \& Challis, P. M. 1997, ApJ, 483,925

Chugai, N. N., \& Danziger, I. J. 1994, MNRAS, 268, 173

Cowan, J. J., \& Branch, D. 1982, ApJ, 258, 31

Cowan, J. J., Goss, W. M., \& Sramek, R. A. 1991, ApJ, 379, L49

DeLaney, T., Rudnick, L., Stage, M. D., et al. 2010, ApJ, 725, 2038

Deneault, E., Clayton, D. D., \& Heger, A. 2003, ApJ, 594, 312

Detre, L., \& Lovas, M. 1970, IAU Circ., 2269, 1

Dwek, E. 2004, ApJ, 607, 848

Elmhamdi, A., Danziger, I. J., Cappellaro, E., et al. 2004, A\&A, 426, 963

Elmhamdi, A., Danziger, I. J., Chugai, N., et al. 2003, MNRAS, 338, 939

Eriksen, K. A., Morse, J. A., Kirshner, R. P., \& Winkler, P. F. 2001, in AIP Conf. Ser. 565, Young Supernova Remnants, ed. S. S. Holt \& U. Hwang (Melville, NY: AIP), 193

Fabian, A. C., Willingale, R., Pye, J. P., Murray, S. S., \& Fabbiano, G. 1980, MNRAS, 193, 175

Feast, M. 1999, in IAU Symp. 190, New Views of the Magellanic Clouds, ed. Y.-H. Chu, N. Suntzeff, J. Hesser, \& D. Bohlender (Cambridge: Cambridge Univ. Press), 542

Fesen, R. A. 1993, ApJ, 413, L109

Fesen, R. A. 2001, ApJS, 133, 161

Fesen, R. A., \& Becker, R. H. 1988, BAAS, 20, 962

Fesen, R. A., \& Becker, R. H. 1990, ApJ, 351, 437

Fesen, R. A., Hurford, A. P., \& Matonick, D. M. 1995, AJ, 109, 2608

Fesen, R. A., \& Matonick, D. M. 1994, ApJ, 428, 157

Fesen, R. A., Morse, J. A., Chevalier, R. A., et al. 2001, AJ, 122, 2644

Fesen, R. A., Gerardy, C. L., Filippenko, A. V., et al. 1999, AJ, 117, 725

Fesen, R. A., Hammell, M. C., Morse, J., et al. 2006, ApJ, 636, 859

Filippenko, A. V., Barth, A. J., Bower, G. C., et al. 1995, AJ, 110, 2261

Filippenko, A. V., \& Matheson, T. 2003, arXiv:0310228

Filippenko, A. V., Matheson, T., \& Barth, A. J. 1994, AJ, 108, 2220

Filippenko, A. V., Matheson, T., \& Ho, L. C. 1993a, ApJ, 415, L103

Filippenko, A. V., Matheson, T., \& Woosley, S. E. 1993b, IAU Circ., 5787, 1

Filippenko, A. V., Treffers, R. R., Paik, Y., Davis, M., \& Schlegel, D. 1993c, IAU Circ., 5731

Fox, O., Skrutskie, M. F., Chevalier, R. A., et al. 2009, ApJ, 691, 650

Fransson, C. 1984, A\&A, 133, 264

Fransson, C., Challis, P. M., Chevalier, R. A., et al. 2005, ApJ, 622, 991

Freedman, W. L., Hughes, S. M., Madore, B. F., et al. 1994, ApJ, 427, 628

Freedman, W. L., Madore, B. F., Gibson, B. K., et al. 2001, ApJ, 553, 47

Garnavich, P. M., \& Ann, H. B. 1993, in BAAS, 25, 1244

Gates, H. S., \& Carpenter, E. F. 1958, Harvard Announcement Card, No., 1394,

Gerardy, C. L., Fesen, R. A., Höflich, P., \& Wheeler, J. C. 2000, AJ, 119, 2968

Goodrich, R. W., Stringfellow, G. S., Penrod, G. D., \& Filippenko, A. V. 1989, ApJ, 342, 908

Hurford, A. P., \& Fesen, R. A. 1996, ApJ, 469, 246

Inserra, C., Turatto, M., Pastorello, A., et al. 2011, MNRAS, 417, 261

Karachentsev, I. D., Sharina, M. E., \& Huchtmeier, W. K. 2000, A\&A, 362, 544

Kasen, D., \& Bildsten, L. 2010, ApJ, 717, 245

Kirshner, R. P. 1990, in Supernovae, ed. A. G. Petschek (New York: SpringerVerlag), 59

Kirshner, R. P., \& Blair, W. P. 1980, ApJ, 236, 135

Kirshner, R. P., \& Kwan, J. 1974, ApJ, 193, 27

Kirshner, R. P., Oke, J. B., Penston, M. V., \& Searle, L. 1973, ApJ, 185, 303

Kochanek, C. S., Szczygiel, D. M., \& Stanek, K. Z. 2011, ApJ, 737, 76

Kotak, R., Meikle, P., Pozzo, M., et al. 2006, ApJ, 651, L117

Kotak, R., Meikle, W. P. S., Farrah, D., et al. 2009, ApJ, 704, 306

Kozma, C., \& Fransson, C. 1998a, ApJ, 496, 946

Kozma, C., \& Fransson, C. 1998b, ApJ, 497, 431

Krause, O., Birkmann, S. M., Rieke, G. H., et al. 2004, Nature, 432, 596

Krause, O., Birkmann, S. M., Usuda, T., et al. 2008, Science, 320, 1195

Kunkel, W., Madore, B., Shelton, I., et al. 1987, IAU Circ., 4316, 1

Lagage, P. O., Claret, A., Ballet, J., et al. 1996, A\&A, 315, L273

Larsson, J., Fransson, C., Östlin, G., et al. 2011, Nature, 474, 484

Lawrence, S. S., MacAlpine, G. M., Uomoto, A., et al. 1995, AJ, 109, 2635

Leibundgut, B., Kirshner, R. P., Pinto, P. A., et al. 1991, ApJ, 372, 531

Leibundgut, B., Kirshner, R. P., \& Porter, A. C. 1993, BAAS, 25, 834

Long, K. S., Blair, W. P., \& Krzeminski, W. 1989, ApJ, 340, L25

Long, K. S., Winkler, P. F., \& Blair, W. P. 1992, ApJ, 395, 632

Massey, P., \& Gronwall, C. 1990, ApJ, 358, 344

Matheson, T., Filippenko, A. V., Barth, A. J., et al. 2000a, AJ, 120, 1487
Matheson, T., Filippenko, A. V., Ho, L. C., Barth, A. J., \& Leonard, D. C. 2000b, AJ, 120, 1499

Matheson, T., Kirshner, R. P., Challis, P., et al. 2008, AJ, 135, 1598

Maund, J. R., \& Smartt, S. J. 2009, Science, 324, 486

Maund, J. R., Smartt, S. J., Kudritzki, R. P., Podsiadlowski, P., \& Gilmore, G. F. 2004, Nature, 427, 129

McCray, R. 1993, ARA\&A, 31, 175

Meikle, W. P. S., Mattila, S., Pastorello, A., et al. 2007, ApJ, 665, 608

Milisavljevic, D., \& Fesen, R. A. 2008, ApJ, 677, 306

Milisavljevic, D., Fesen, R. A., Kirshner, R. P., \& Challis, P. 2009, ApJ, 692, 839

Milisavljevic, D., Fesen, R. A., Leibundgut, B., \& Kirshner, R. P. 2008, ApJ, 684, 1170

Montes, M. J., van Dyk, S. D., Weiler, K. W., Sramek, R. A., \& Panagia, N. 1997, ApJ, 482, L61

Montes, M. J., van Dyk, S. D., Weiler, K. W., Sramek, R. A., \& Panagia, N. 1998, ApJ, 506, 874

Morse, J. A., Winkler, P. F., \& Kirshner, R. P. 1995, AJ, 109, 2104

Nymark, T. K., Chandra, P., \& Fransson, C. 2009, A\&A, 494, 179

Patnaude, D. J., Loeb, A., \& Jones, C. 2011, New Astron., 16, 187

Pennington, R. L., \& Dufour, R. J. 1983, ApJ, 270, L7

Pennington, R. L., Talbot, R. J., Jr., \& Dufour, R. J. 1982, AJ, 87, 1538

Reed, J. E., Hester, J. J., Fabian, A. C., \& Winkler, P. F. 1995, ApJ, 440, 706

Rest, A., Foley, R. J., Sinnott, B., et al. 2011, ApJ, 732, 3

Rho, J., Kozasa, T., Reach, W. T., et al. 2008, ApJ, 673, 271

Richmond, M. W., Treffers, R. R., Filippenko, A. V., et al. 1994, AJ, 107, 1022

Ripero, J., Garcia, F., Rodriguez, D., et al. 1993, IAU Circ., 5731, 1

Ryder, S., Staveley-Smith, L., Dopita, M., et al. 1993, ApJ, 416, 167

Saha, A., Thim, F., Tammann, G. A., Reindl, B., \& Sandage, A. 2006, ApJS, 165,108

Schlegel, E. M. 1994, AJ, 108, 1893

Schlegel, E. M., Ryder, S., Staveley-Smith, L., et al. 1999, AJ, 118, 2689

Seaquist, E. R., \& Bignell, R. C. 1978, ApJ, 226, L5

Smith, J. D. T., Rudnick, L., Delaney, T., et al. 2009, ApJ, 693, 713

Smith, N., Foley, R. J., \& Filippenko, A. V. 2008, ApJ, 680, 568

Soria, R., \& Perna, R. 2008, ApJ, 683, 767

Stockdale, C. J., Goss, W. M., Cowan, J. J., \& Sramek, R. A. 2001, ApJ, 559, L139

Stone, R. P. S. 1977, ApJ, 218, 767

Stringfellow, G. S., Goodrich, R. W., Filippenko, A. V., \& Penrod, G. D. 1988, BAAS, 20, 962

Sugerman, B. E. K., Andrews, J. E., Barlow, M. J., et al. 2012, ApJ, 749, 170

Sugerman, B. E. K., Ercolano, B., Barlow, M. J., et al. 2006, Science, 313, 196 Suzuki, T., \& Nomoto, K. 1995, ApJ, 455, 658

Thorstensen, J. R., Fesen, R. A., \& van den Bergh, S. 2001, AJ, 122, 297

Tuohy, I. R., \& Dopita, M. A. 1983, ApJ, 268, L11

Turatto, M., Cappellaro, E., Barbon, R., et al. 1990, AJ, 100, 771

Turatto, M., Cappellaro, E., \& Danziger, I. J. 1989, Messenger, 56, 36

Uomoto, A. 1991, AJ, 101, 1275

Uomoto, A., \& Kirshner, R. P. 1986, ApJ, 308, 685

van den Bergh, S. 1988, ApJ, 327, 156

van Dyk, S. D., Hamuy, M., \& Filippenko, A. V. 1996, AJ, 111, 2017

van Dyk, S. D., Weiler, K. W., Sramek, R. A., Rupen, M. P., \& Panagia, N. 1994, ApJ, 432, L115

van Leeuwen, F., Feast, M. W., Whitelock, P. A., \& Laney, C. D. 2007, MNRAS, 379,723

Vink, J., Kaastra, J. S., \& Bleeker, J. A. M. 1996, A\&A, 307, L41

Vogt, F., \& Dopita, M. A. 2010, ApJ, 721, 597

Vogt, F., \& Dopita, M. A. 2011, Ap\&SS, 331, 521

Weiler, K. W., Panagia, N., Montes, M. J., \& Sramek, R. A. 2002, ARA\&A, 40, 387

Weiler, K. W., Sramek, R. A., Panagia, N., van der Hulst, J. M., \& Salvati, M. 1986, ApJ, 301, 790

Weiler, K. W., van Dyk, S. D., Panagia, N., \& Sramek, R. A. 1992, ApJ, 398, 248

Weiler, K. W., Williams, C. L., Panagia, N., et al. 2007, ApJ, 671, 1959

Winzer, J. E. 1974, J. R. Astron. Soc. Can., 68, 36

Woosley, S. E. 2010, ApJ, 719, L204

Woosley, S. E., Eastman, R. G., Weaver, T. A., \& Pinto, P. A. 1994, ApJ, 429, 300

Woosley, S. E., Pinto, P. A., Martin, P. G., \& Weaver, T. A. 1987, ApJ, 318, 664

Young, P. A., Fryer, C. L., Hungerford, A., et al. 2006, ApJ, 640, 891

Young, T. R., \& Branch, D. 1989, ApJ, 342, L79

Zimmermann, H.-U., Lewin, W., Predehl, P., et al. 1994, Nature, 367, 621 